Moroccan J. of Pure and Appl. Anal. (MJPAA)

Volume 5(1), 2019, Pages 1-21

ISSN: Online 2351-8227 - Print 2605-6364

DOI 10.2478/mjpaa-2019-0001

\title{
On some parabolic problems with measure sources
}

\author{
MoHAMMEd ABDELLAOUI ${ }^{1, a}$
}

AвSTRACт. One of the recent advances in the investigation of nonlinear parabolic equations with a measure as forcing term is a paper by F. Petitta in which it has been introduced the notion of renormalized solutions to the initial parabolic problem in divergence form. Here we continue the study of the stability of renormalized solutions to nonlinear parabolic equations with measures but from a different point of view: we investigate the existence and uniqueness of the following nonlinear initial boundary value problems with absorption term and a possibly sign-changing measure data

$$
\begin{cases}b(u)_{t}-\operatorname{div}(a(t, x, u, \nabla u))+h(u)=\mu & \text { in } Q:=(0, T) \times \Omega, \\ u=0 & \text { on }(0, T) \times \partial \Omega, \\ b(u)=b\left(u_{0}\right) & \text { in } \Omega,\end{cases}
$$

where $\Omega$ is an open bounded subset of $\mathbb{R}^{N}, N \geq 2, T>0$ and $Q$ is the cylinder $(0, T) \times \Omega, \Sigma=(0, T) \times \partial \Omega$ being its lateral surface, the operator is modeled on the $p$-Laplacian with $p>2-\frac{1}{N+1}, \mu$ is a Radon measure with bounded total variation on $Q, b$ is a $C^{1}$-increasing function which satisfies $0<b_{0} \leq b^{\prime}(s) \leq b_{1}$ (for positive constants $b_{0}$ and $\left.b_{1}\right)$. We assume that $b\left(u_{0}\right)$ is an element of $L^{1}(\Omega)$ and $h: \mathbb{R} \mapsto \mathbb{R}$ is a continuous function such that $h(s) s \geq 0$ for every $|s| \geq L$ and $L \geq 0$ (odd functions for example). The existence of a renormalized solution is obtained by approximation as a consequence of a stability result. We provide a new proof of this stability result, based on the properties of the truncations of renormalized solutions. The approach, which does not need the strong convergence of the truncations of the solutions in the energy space, turns out to be easier and shorter than the original one.

2010 Mathematics Subject Classification. Primary 35K67, 37K45, 35A35, 35S05, 28A12

Key words and phrases. Generalized solutions, Measures and capacities, Nonlinear parabolic equations, Pseudo-monotone operators, Stability.

Received May 05, 2019 - Accepted June 09, 2019.

(C) The Author(s) 2017. This article is published with open access by Sidi Mohamed Ben Abdallah University.

${ }^{1}$ University of Fez, Faculty of Sciences Dhar El Mahraz, Laboratory LAMA, Department of Mathematics, B.P. 1796, Atlas Fez, Morocco.

a e-mail: mohammed.abdellaoui3@usmba.ac.ma . 


\section{Introduction}

First, let us consider the nonlinear parabolic problem

$$
\left\{\begin{array}{l}
u_{t}-\operatorname{div}(a(t, x, u, \nabla u))=\mu \text { in } Q:=(0, T) \times \Omega, \\
u=0 \text { on }(0, T) \times \partial \Omega, u(0)=u_{0} \text { in } \Omega
\end{array}\right.
$$

where $\Omega$ is an open bounded subset of $\mathbb{R}^{N}, N \geq 2, T>0$ and $Q$ is the cylinder $(0, T) \times \Omega$, the operator of Leray-Lions $u \mapsto-\operatorname{div}(a(t, x, u, \nabla u))$ is pseudo-monotone defined on the space $L^{p}\left(0, T ; W_{0}^{1, p}(\Omega)\right)$ with values in its dual $L^{p^{\prime}}\left(0, T ; W^{-1, p^{\prime}}(\Omega)\right), p>1$ and $\frac{1}{p}+\frac{1}{p^{\prime}}=1$. Recall that the hypothesis on $p$ (i.e., $\left.p>2-\frac{1}{N+1}\right)$ is motivated by the fact that, if $p \leq 2-\frac{1}{N+1}$ then $\frac{N p+p-N}{N+1} \leq 1$ this implies that, in order to obtain the existence of a solution for $p$ close to 1, it is necessary to go out of the framework of classical Sobolev spaces (see [DMOP, Example 2.16] that can be easily extended in parabolic case). It is well known that, if the datum $\mu$ belongs to $L^{p^{\prime}}(Q)$, the parabolic problem (1.1) admits a unique variational solution $u \in L^{p}\left(0, T ; W_{0}^{1, p}(\Omega)\right)$ and to $C\left(0, T ; L^{2}(\Omega)\right)$ (see [L1, L2]). In the general case where the datum $\mu$ is a Radon measure with bounded variation on $Q$, it is known that there exist at least one solution to (1.1) in the sense of distributions [BDGO, BG]. As a matter of fact, this notion of weak solutions does not guarantee the uniqueness, even for linear equations [Pr1, Se]. The uniqueness of the solutions can be obtained in the linear case for general data, using the definition by duality [S], this Stammpacchia's framework heavily concern the case $p=2$ and $a(x, \nabla u)=A(x) \nabla u$ where $A$ is a uniformly elliptic matrix with $L^{\infty}(\Omega)$-coefficients is extended in parabolic case when the linear operator is strongly monotone, Lipschitz continuous with respect to $\nabla u$ and the datum $\mu$ belongs to $\mathcal{M}_{0}(Q)$ in $[\mathrm{Pe} 1$, Section 9] and continue to work for general measure data in $\mathcal{M}_{b}(Q)$ (see Petitta's ideas [Pe3]). These results are generalized in the nonlinear case and for measures in $L^{1}$-data, in $L^{1}(\Omega)+W^{-1, p^{\prime}}(\Omega)$ or in $\mathcal{M}_{0}(Q)$ for bounded measure on $Q$ that does not charges the sets of zero parabolic $p$-capacity using the notion of renormalized solution in [BM, DP, DPP] and using the notion of entropy solution in [Pr2]. In [Pe1, PP] it was introduced and studied a notion of renormalized solution for general measure data: the key observation is that every Radon measure $\mu$ with bounded variation can be decomposed as $\mu=\mu_{0}+\mu_{s}, \mu_{0} \in \mathcal{M}_{0}(\Omega), \mu_{s} \in \mathcal{M}_{s}(Q)$ (see $[\mathrm{Pe} 1$, Lemma 2.1]). In addition, a measure $\mu$ belongs to $\mathcal{M}_{0}(Q)$ if, see [DPP, Theorem 1.1] and [PPP1, Proposition 1.1], and only if [KR, Theorem 2.6], it belongs to $L^{1}(Q)+L^{p^{\prime}}\left(0, T ; W^{-1, p^{\prime}}(\Omega)\right)+L^{p}(0, T ; V)$, while $\mu_{s}$ is concentrated on a set of $p$-capacity zero. Then, it is reasonable to assume that a good definition should imply that the solution has a very large absolute value only where the datum is very concentrated, that is on the support of $\mu_{s}$. Finally, the renormalized solution is a special type of solutions in the sense of distribution. The definition proposed in [Pe1] is the following: a renormalized solution to (1.1) is a measurable function $u$ with all translations $u-g$ in the space $L^{q}\left(0, T ; W_{0}^{1, q}(\Omega)\right) \cap L^{\infty}\left(0, T ; L^{1}(\Omega)\right)$ for every $q<p-\frac{N}{N+1}$ and all truncations $T_{k}(u-g)$ in the energy space $L^{p}\left(0, T ; W_{0}^{1, p}(\Omega)\right) \cap L^{\infty}\left(0, T ; L^{1}(\Omega)\right)$ and such that for every $S \in W^{2, \infty}(\mathbb{R})(S(0)=0)$ with $S^{\prime}$ has compact support on $\mathbb{R}$, we have

$$
\begin{aligned}
& -\int_{\Omega} S\left(u_{0}\right) \varphi(0) d x-\int_{0}^{T}\left\langle\varphi_{t}, S(u-g)\right\rangle d t+\int_{Q} S^{\prime}(u-g) a(t, x, u, \nabla u) \cdot \nabla \varphi d x d t \\
& \quad+\int_{Q} S^{\prime \prime}(u-g) a(t, x, u, \nabla u) \cdot \nabla(u-g) \varphi d x d t=\int_{Q} S^{\prime}(u-g) \varphi d \tilde{\mu}_{0},
\end{aligned}
$$

for every functions $\varphi \in L^{p}\left(0, T ; W_{0}^{1, p}(\Omega)\right) \cap L^{\infty}(Q), \varphi_{t} \in L^{p^{\prime}}\left(0, T ; W^{-1, p^{\prime}}(\Omega)\right)$, with $\varphi(T, x)=0$, such that $S^{\prime}(u-$ $g) \varphi \in L^{p}\left(0, T ; W_{0}^{1, p}(\Omega)\right), g$ is the time derivative part of $\mu_{0}$ and $\hat{\mu}_{0}=\mu-g_{t}-\mu_{s}=f-\operatorname{div}(G)$. Moreover, for every $\psi \in C(\bar{Q})$ we have

$$
\left\{\begin{array}{l}
\lim _{n \rightarrow+\infty} \frac{1}{n} \int_{\{n \leq v<2 n\}} a(t, x, u, \nabla u) \cdot \nabla v \psi d x d t=\int_{Q} \psi d \mu_{s}^{+}, \\
\lim _{n \rightarrow+\infty} \frac{1}{n} \int_{\{-2 n<v \leq-n\}} a(t, x, u, \nabla u) \cdot \nabla v \psi d x d t=\int_{Q} \psi d \mu_{s}^{-},
\end{array}\right.
$$


where $\mu_{s}^{+}$and $\mu_{s}^{-}$are respectively the positive and the negative parts of the singular term of the measure $\mu$ with respect to the $p$-capacity. The existence of solutions to (1.1) is obtained as a consequence of a stability result. Namely, every Radon measure $\mu=\mu_{0}+\mu_{s}$ can be approximated, essentially by convolutions, by a sequence $\left(\mu_{n}\right)$ of measures in $L^{p^{\prime}}\left(0, T ; W^{-1, p^{\prime}}(\Omega)\right)$. By classical results, for every $n$ there exists a unique variational (and hence renormalized) solution $u_{n}$ of

$$
\left\{\begin{array}{l}
u_{t}^{n}-\operatorname{div}\left(a\left(t, x, u_{n}, \nabla u_{n}\right)\right)=\mu_{n} \text { in } Q:=(0, T) \times \Omega, \\
u_{n}=0 \text { on }(0, T) \times \partial \Omega, u_{n}(0)=u_{0} \text { in } \Omega .
\end{array}\right.
$$

Moreover, thanks to the linearity of the convolutions, the measure $\mu_{n}$ can be split in several terms which have good convergence properties as $n$ goes to $+\infty$. With this model in mind, the approach followed in [Pe1] is to consider sequences $\left(\mu_{n}\right)$ of Radon measures with bounded variation on $Q$ having a splitting which converges to a Radon measure $\mu$ (see Definition 2.2), and to prove that the corresponding renormalized solutions $\left(u_{n}\right)$ converge in suitable topologies to a renormalized solution to (1.1). The proof in [Pe1] is rather technical, and it can be split into two parts. As a first step, the equation solved by $u_{n}$ is used in order to obtain some a priori estimates, and hence a weak limit $u$ of $\left(u_{n}\right)$, which is the candidate to be the solution to (1.1). In particular it is easily proved that, up to a subsequence, every truncation $T_{k}\left(u_{n}\right)$ converges to the corresponding truncation $T_{k}(u)$ in the weak topology of $L^{p}\left(0, T ; W_{0}^{1, p}(\Omega)\right)$. The second part, which is the hardest one, is devoted to showing that the sequence of truncations converges, in fact, in the strong topology of $L^{p}\left(0, T ; W_{0}^{1, p}(\Omega)\right)$.

In this paper, the starting point will be the end of the first point of the proof in [Pe1] (the a priori estimates), and the goal will be to pass to the limit in $n$ using the equation solved by the truncations of $u_{n}$ (see Definition 2.3). The major advantage of this approach is that we can perform the passage to the limit without using the strong convergence of the truncations in $L^{p}\left(0, T ; W_{0}^{1, p}(\Omega)\right)$.

\section{Preliminaries}

2.1. Notations. Throughout this paper $\Omega$ will be a bounded open subset of $\mathbb{R}^{N}, N \geq 2, p$ and $p^{\prime}$ will be real numbers with $p>1$ and $\frac{1}{p}+\frac{1}{p^{\prime}}=1$. The duality pairing between the Sobolev space $W_{0}^{1, p}(\Omega)$ and its dual $W^{-1, p^{\prime}}(\Omega)$ will be denoted by $\langle\cdot, \cdot\rangle$. In what follows, $|\zeta|$ and $\zeta \cdot \zeta^{\prime}$ will denote respectively the Euclidean norm of a vector $\zeta \in \mathbb{R}^{N}$ and the scalar product between $\zeta$ and $\zeta^{\prime} \in \mathbb{R}^{N}$. The characteristic function of a set $E \subseteq \mathbb{R}^{N}$ will be defined as

$$
\chi_{E}= \begin{cases}1, & x \in E, \\ 0, & x \notin E\end{cases}
$$

2.2. The operator. Let $a:(0, T) \times \Omega \times \mathbb{R} \times \mathbb{R}^{N} \mapsto \mathbb{R}^{N}$ be Carathéodory function (that is, $a(\cdot, \cdot, s, \zeta)$ is measurable on $Q$ for every $(s, \zeta)$ in $\mathbb{R} \times \mathbb{R}^{N}$ and $a(t, x, \cdot, \cdot)$ is continuous on $\mathbb{R} \times \mathbb{R}^{N}$ for almost every $(t, x)$ in $\left.Q\right)$. Assume that there exist two positive constants $c_{0}, c_{1}$, and a nonnegative function $b_{0}(t, x) \in L^{p^{\prime}}(Q)$, such that, for every $\zeta, \zeta^{\prime} \in \mathbb{R}^{N}$ with $\zeta \neq \zeta^{\prime}$, the following properties hold

$$
\begin{gathered}
a(t, x, s, \zeta) \cdot \zeta \geq c_{0}|\zeta|^{p}, \\
|a(t, x, s, \zeta)| \leq b_{0}(t, x)+c_{1}\left(|s|^{p-1}+|\zeta|^{p-1}\right), \\
(a(t, x, s, \zeta)-a(t, x, s, \eta)) \cdot\left(\zeta-\zeta^{\prime}\right)>0 .
\end{gathered}
$$

Thanks to hypotheses $(2.1)-(2.3)$, the map $u \mapsto-\operatorname{div}(a(t, x, u, \nabla u))$ is a coercive, continuous, bounded and monotone operator defined on $L^{p}\left(0, T ; W_{0}^{1, p}(\Omega)\right)$ with values in $L^{p^{\prime}}\left(0, T ; W^{-1, p^{\prime}}(\Omega)\right)$; hence by the standard theory of monotone operators (see, e.g., [L1]), for every $F$ in $L^{p^{\prime}}(Q)$ and $u_{0} \in L^{2}(\Omega)$ there exists a variational solution $u$ of the problem

$$
\left\{\begin{array}{l}
u_{t}-\operatorname{div}(a(t, x, u, \nabla u))=F \text { in } Q:=(0, T) \times \Omega, \\
u=0 \text { on }(0, T) \times \partial \Omega, \quad u(0)=u_{0} \text { in } \Omega,
\end{array}\right.
$$




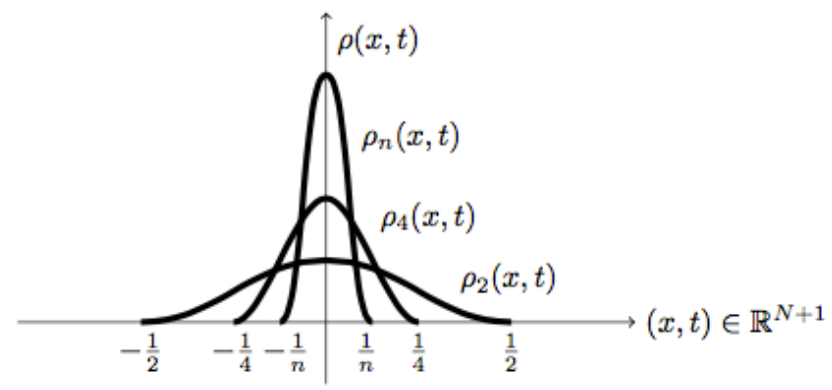

FIGURE 1. Example of a mollifier

in the sense that $u$ belongs to $W \cap C\left(0, T ; L^{2}(\Omega)\right.$ ) (where $W=\left\{u \in L^{p}(0, T ; V), u_{t} \in L^{p^{\prime}}\left(0, T ; V^{\prime}\right)\right\}$ with $V=$ $\left.W_{0}^{1, p}(\Omega) \cap L^{2}(\Omega)\right)$, and

$$
-\int_{\Omega} u_{0} \varphi(0) d x-\int_{0}^{T}\left\langle\varphi_{t}, v\right\rangle d t+\int_{Q} a(t, x, v, \nabla v) \cdot \nabla \varphi d x d t=\int_{0}^{T}\langle F, \varphi\rangle_{W^{-1, p^{\prime}}(\Omega), W_{0}^{1, p}(\Omega)} d t,
$$

for all $\varphi \in W$ such that $\varphi(T)=0$.

Remark 2.1. Note that, the entropy solution $u$ of problem (1.1) exists and is unique as shown in [DPP] if $\mu \in \mathcal{M}_{0}(Q)$ (see also [DP]). Moreover, the solution is such that $|a(t, x, u, \nabla u)| \in L^{q}(Q)$ for all $q<1+\frac{1}{(N+1)(p-1)}$, even if its gradient may not belong to any Lebesgue space, this notion can not be generalized directly to the case of a general, possibly singular, measure in $\mathcal{M}_{b}(Q)$.

Definition 2.1. A sequence of measures $\left(\mu_{n}\right)$ in $Q$ is equi-diffuse if $\left(\mu_{n}\right)$ is bounded in $\mathcal{M}_{b}(Q)$ and for every $\epsilon>0$ there exists $\eta>0$ such that

$$
\operatorname{cap}_{p}(E)<\eta \Rightarrow\left|\mu_{n}\right|(E)<\epsilon \quad \forall n \geq 1 .
$$

Proposition 2.1. Let $\mu \in \mathcal{M}_{0}(Q)$ with compact support and let $\left(\rho_{n}\right)$ be a sequence of mollifiers. Then $\left(\rho_{n} * \mu\right)$ is equidiffuse.

2.3. Capacity. For every Borel set $B \subseteq Q$, its $p$-capacity $\operatorname{cap}_{p}(B, Q)$ with respect to $Q$ is defined by

$$
\operatorname{cap}_{p}(B, Q)=\inf \left\{\|u\|_{W}\right\}
$$

where the infimum is taken over all the functions $u \in W$ such that $u \geq 1$ almost everywhere in a neighborhood of $B$. We say that a property $\mathcal{P}(t, x)$ holds cap $_{p}$ - quasi everywhere if $\mathcal{P}(t, x)$ holds for every $(t, x)$ outside a subset of $Q$ of zero $p$-capacity. A function $u$ defined on $Q$ is said to be $\operatorname{cap}_{p}$-quasi continuous if for every $\epsilon>0$ there exists $B \subseteq Q$ with $\operatorname{cap}_{p}(B, Q)<\epsilon$ such that the restriction of $u$ to $Q \backslash B$ is continuous. It is well known that every function in $W$ has a unique $\operatorname{cap}_{p}$-quasi continuous representative, whose values are defined $\operatorname{cap}_{p}-$ quasi everywhere in $Q$ (see [DPP, Pe1]). In what follows we always identify a function $u \in W$ with its cap ${ }_{p}-$ quasi continuous representative. A set $E \subseteq Q$ is said to be cap $_{p}$-quasi open if for every $\epsilon>0$ there exists an open set $U$

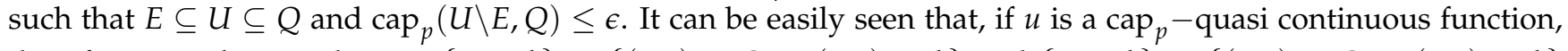
then for every $k \in \mathbb{R}$, the sets $\{u>k\}=\{(t, x) \in Q: u(t, x)>k\}$ and $\{u<k\}=\{(t, x) \in Q: u(t, x)<k\}$ are $\operatorname{cap}_{p}$-quasi open. The characteristic function of a cap casi $_{p}$-quasi set can be approximated by a monotonic sequence of functions in the energy space $W$, as stated in the following Lemma (see [PPP2, Lemma 2.1]).

Lemma 2.1. For every cap $p_{p}$-quasi open set $E \subseteq Q$ there exists an increasing sequence $\left(w_{n}\right)$ of nonnegative functions in $W$ which converges to $\chi_{E}$ cap $p_{p}-$ quasi everywhere in $Q$. 
2.4. Truncations. For every $k \in \mathbb{R}^{+}=(0,+\infty)$, we define the truncation function $T_{k}: \mathbb{R} \mapsto \mathbb{R}$ by

$$
T_{k}(s)=\max (-k, \min (k, s)), \quad s \in \mathbb{R} \text {. }
$$

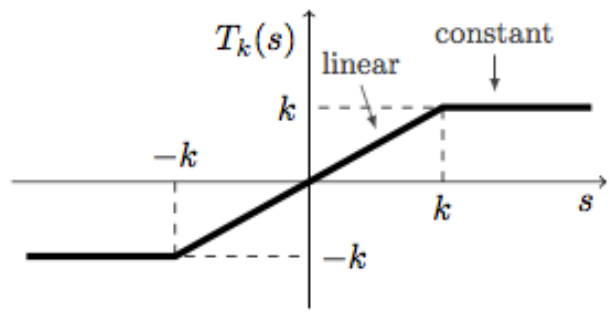

Figure 2. The function $T_{k}(s)$

Let us consider the space of all measurable functions, finite a.e. in $Q$, such that $T_{k}(u)$ belongs to $L^{p}\left(0, T ; W_{0}^{1, p}(\Omega)\right)$ for every $k>0$. It is easy to see that every function $u$ in this space has a cap ${ }_{p}$-quasi continuous representative, that will always be identified with $u$. Moreover, there exists a measurable function $v: Q \mapsto \mathbb{R}^{N}$ such that $\nabla T_{k}(u)=v \chi_{\{|u|<k\}}$ a.e. in $Q$, for every $k>0$, and $v$ is unique up to almost everywhere equivalence (see [B6, Lemma 2.1]). Hence it is possible to define a generalized gradient $\nabla u$ of $u$, setting $\nabla u=v$. If $u \in L^{1}\left(0, T ; W_{0}^{1,1}(\Omega)\right)$, this gradient coincides with the usual one, while for $u \in L^{1}\left(0, T ; L_{l o c}^{1}(\Omega)\right)$, it may differ from the usual distributional gradient of $u$.

2.5. Measures. We define $\mathcal{M}_{b}(Q)$ as the space of all Radon measures on $Q$ with bounded total variation, and $C_{b}(Q)$ as the space of all bounded, continuous functions on $Q$, so that $\int_{Q} \varphi d \mu$ is defined for $\varphi \in C_{b}(Q)$ and $\mu$ in $\mathcal{M}_{b}(Q)$. The positive, the negative and the total variation parts of a measure $\mu$ in $\mathcal{M}_{b}(Q)$ are denoted by $\mu^{+}$, $\mu^{-}$, and $|\mu|$, respectively. Given a measure $\mu$ in $\mathcal{M}_{b}(Q)$, and a Borel set $E \subseteq Q$, the measure $\mu\llcorner E$ is defined by $\left(\mu\llcorner E)(Q)=\mu(E \cap B)\right.$ for any Borel set $B \subseteq Q$. We say that a sequence $\left(\mu_{n}\right)$ of measures in $\mathcal{M}_{b}(Q)$ converges tightly (or, equivalently, in the narrow topology of measures) to a measure $\mu$ in $\mathcal{M}_{b}(Q)$ if

$$
\lim _{n \rightarrow+\infty} \int_{Q} \varphi d \mu_{n}=\int_{Q} \varphi d \mu
$$

for every $\varphi \in C_{b}(Q)$. If (2.5) holds only for all the continuous functions $\varphi$ with compact support in $Q$, then we have the usual weak* convergence in $\mathcal{M}_{b}(Q)$ (We point out that, at least for nonnegative measures, tight convergence is equivalent to weak* convergence provided the masses converge, that is, $\mu_{n}$ converges tightly to $\mu$ is and only if $\mu_{n}$ converges to $\mu$ weakly* in $\mathcal{M}_{b}(Q)$ and $\mu_{n}(Q)$ converges to $\left.\mu(Q)\right)$.

We define $\mathcal{M}_{0}(Q)$ as the set of all measures $\mu$ in $\mathcal{M}_{b}(Q)$ which satisfy $\mu(B)=0$ for every Borel set $B \subseteq Q$ such that $\operatorname{cap}_{p}(B, Q)=0$, while $\mathcal{M}_{s}(Q)$ will be the set of all measures in $\mathcal{M}_{b}(Q)$ for which there exists a Borel set $E \subset Q$, with $\operatorname{cap}_{p}(E, Q)=0$, such that $\mu=\mu\llcorner E$.

In order to localize some integrals near the support of the singular measure $\mu_{s}$ with respect to $p$-capacity. Let us consider the space

$$
S=\left\{u \in L^{p}\left(0, T ; W_{0}^{1, p}(\Omega)\right) ; u_{t} \in L^{p^{\prime}}\left(0, T ; W^{-1, p^{\prime}}(\Omega)\right)+L^{1}(Q)\right\},
$$

endowed with its natural norm $\|u\|_{S}=\|u\|_{L^{p}\left(0, T ; W_{0}^{1, p}(\Omega)\right)}+\left\|u_{t}\right\|_{L^{p^{\prime}}\left(0, T ; W^{-1, p^{\prime}}(\Omega)\right)+L^{1}(Q)}$ and used to estimate the cut-off functions in order to treat the singular term of the measure. The cap $p_{p}$-quasi continuous representative of a function in $W \cap L^{\infty}(Q)$ has good integrability properties w.r.t a measure in $\mathcal{M}_{0}(Q)$ as stated in the following result

Proposition 2.2. Let $\mu_{0}$ be a measure in $\mathcal{M}_{0}(Q)$ and let $u$ be a function in $W$. Then $u$ is measurable with respect to $\mu_{0}$. If $u$ further is in $L^{\infty}(Q)$, then $u$ belongs to $L^{\infty}\left(Q, \mu_{0}\right)$, hence to $L^{1}\left(Q, \mu_{0}\right)$, and $\|u\|_{L^{\infty}\left(Q, \mu_{0}\right)}=\|u\|_{L^{\infty}(Q)}$. 
Proof. The proof can be performed arguing as in [DMOP, HKM] in the elliptic case.

An easy consequence of Proposition 2.2 and of the Dominated Convergence Theorem is the following result

Proposition 2.3. Let $\mu_{0}$ be a measure in $\mathcal{M}_{0}(Q)$ and let $\left(u_{n}\right)$ be a sequence of functions in $W \cap L^{\infty}(Q)$, bounded in $L^{\infty}(Q)$ and converging to a function $u$ cap $p_{p}$-quasi everywhere. Then $\left(u_{n}\right)$ converges to $u \mu_{0}$-almost everywhere, and

$$
\lim _{n \rightarrow \infty} \int_{Q} u_{n} d \mu_{0}=\int_{Q} u d \mu_{0} .
$$

The structure of a measure belonging to $\mathcal{M}_{0}(Q)$ is described by the following theorem

Theorem 2.1. If $\mu_{0}$ is a measure in $\mathcal{M}_{0}(Q)$ then there exist $f \in L^{1}(Q),-\operatorname{div}(G) \in L^{p^{\prime}}\left(0, T ; W^{-1, p^{\prime}}(\Omega)\right)$ and $g \in$ $L^{p}(0, T ; V)$ such that $\mu_{0}=f-\operatorname{div}(G)+g_{t}$ in the sense of distributions. Moreover

$$
\int_{Q} \varphi d \mu=\int_{Q} f \varphi d x d t+\int_{0}^{T}\langle-\operatorname{div}(G), \varphi\rangle d t-\int_{0}^{T}\left\langle\varphi_{t}, g\right\rangle d t .
$$

for any $\varphi \in C_{c}^{\infty}([0, T] \times \Omega)$, where $\langle\cdot, \cdot\rangle$ denotes the duality between $V^{\prime}$ and $V$.

Proof. See [DPP, Theorem 1.1].

Note that the reverse of Theorem 2.1 is also true, that is, if $\mu_{0}$ have a decomposition $(f, G, g)$ then it is diffuse (see [PPP1, Example 1] and [PPP2, Proposition 3.1] for $g \in L^{\infty}(Q)$ and [KR, Theorem 2.6] for any conditions on $g)$. On the other hand, every measure in $\mathcal{M}_{b}(Q)$ admits a decomposition as follows

Theorem 2.2. If $\mu \in \mathcal{M}_{b}(Q)$, then there exists a unique pair $\left(\mu_{0}, \mu_{s}\right)$ such that $\mu=\mu_{0}+\mu_{s}, \mu_{0} \in \mathcal{M}_{0}(Q)$ and $\mu_{s} \in \mathcal{M}_{s}(Q)$.

Proof. See [FST, Lemma 2.1].

In conclusion, every measure $\mu \in \mathcal{M}_{b}(Q)$ can be decomposed (not in a unique way) as

$$
\mu=f-\operatorname{div}(G)+g_{t}+\mu_{s}^{+}-\mu_{s}^{-},
$$

with $f \in L^{1}(Q),-\operatorname{div}(G) \in L^{p^{\prime}}\left(0, T ; W^{-1, p^{\prime}}(\Omega)\right), g \in L^{p}(0, T ; V)$ and $\mu_{s} \in \mathcal{M}_{s}(Q)$.

Fixed $\mu \in \mathcal{M}_{b}(Q)$, we consider a special type of approximating sequence $\left(\mu_{n}\right)$ as in [Pe1] and defined as follows

Definition 2.2. Let $\mu \in \mathcal{M}_{b}(Q)$ be decomposed as $\mu=f-\operatorname{div}(G)+g_{t}+\mu_{s}^{+}-\mu_{s}^{-}$, and let $\left(\mu_{n}\right)$ be a sequence of measures in $\mathcal{M}_{b}(Q)$. We say that $\left(\mu_{n}\right)$ has a splitting $\left(f_{n}, G_{n}, g_{n}, \lambda_{n}^{\oplus}, \lambda_{n}^{\ominus}\right)$ converging to $\mu$ as $n$ tends to $+\infty$, if for every $n$ the measure $\mu_{n}$ can be decomposed as

$$
\mu_{n}=f_{n}-\operatorname{div}\left(G_{n}\right)+g_{n}+\lambda_{n}^{\oplus}-\lambda_{n}^{\ominus},
$$

and the following properties hold:

(i) $\left(f_{n}\right)$ is a sequence of functions in $L^{1}(Q)$ that converges to $f$ weakly in $L^{1}(Q)$;

(ii) $\left(G_{n}\right)$ is a sequence of functions in $\left(L^{p^{\prime}}(Q)\right)^{N}$ that converges to $G$ strongly in $\left(L^{p^{\prime}}(Q)\right)^{N}$, and such that $\left(-\operatorname{div}\left(G_{n}\right)\right)$ is bounded in $\mathcal{M}_{b}(Q)$;

(iii) $\left(g_{n}\right)$ is a sequence of functions in $L^{p}\left(0, T ; W_{0}^{1, p}(\Omega)\right)$ that converges to $g$ strongly in $L^{p}\left(0, T ; W_{0}^{1, p}(\Omega)\right)$;

(iv) $\left(\lambda_{n}^{\oplus}\right)$ is a sequence of nonnegative measures in $\mathcal{M}_{b}(Q)$ that converges to $\mu_{s}^{+}$in the narrow topology of measures;

(v) $\left(\lambda_{n}^{\ominus}\right)$ is a sequence of nonnegative measures in $\mathcal{M}_{b}(Q)$ that converges to $\mu_{s}^{-}$in the narrow topology of measures.

Remark 2.2. It is easy to see that, if $\left(\mu_{n}\right)$ has a splitting converging to $\mu$, then $\left(\mu_{n}\right)$ converges weakly* in $\mathcal{M}_{b}(Q)$ to $\mu$, so that there exists $M>0$ such that $\left|\mu_{n}(Q)\right| \leq M$ for every $n$.

Remark 2.3. From the definition of the splitting we have that the part $\mu_{n, 0}$ of $\mu_{n}$ absolutely continuous with respect to the $p$-capacity is given by

$$
\mu_{n, 0}=f_{n}-\operatorname{div}\left(G_{n}\right)+g_{n}+\lambda_{n, 0}^{\oplus}-\lambda_{n, 0}^{\ominus} .
$$

On the other hand, the singular part of $\mu_{n}$ can be estimated by

$$
\mu_{n, s}^{+} \leq \lambda_{n, s}^{\oplus} \quad \mu_{n, s}^{-} \leq \lambda_{n, s}^{\ominus} .
$$


Remark 2.4. If we decompose the measures $\mu_{n}, \lambda_{n}^{\oplus}, \lambda_{n}^{\ominus}$, respectively as, $\mu_{n}=\mu_{\epsilon, 0}+\mu_{n, s}, \lambda_{n}^{\oplus}=\lambda_{n, 0}^{\oplus}+\lambda_{n, s}^{\oplus}\left(\lambda_{n, 0}^{\oplus}=\right.$ $\left.\lambda_{n, 0}^{1, \oplus}-\operatorname{div}\left(\lambda_{n, 0}^{2, \oplus}\right)\right), \lambda_{n}^{\ominus}=\lambda_{n, 0}^{\ominus}+\lambda_{n, s}^{\ominus}\left(\lambda_{n, 0}^{\ominus}=\lambda_{n, 0}^{1, \ominus}-\operatorname{div}\left(\lambda_{n, 0}^{2, \ominus}\right)\right)$, with $\mu_{n, 0}, \lambda_{n, 0}^{\oplus}$ and $\lambda_{n, 0}^{\ominus}$ in $\mathcal{M}_{0}(Q)$, and $\mu_{n, s}, \lambda_{n, s}^{\oplus}$ and $\lambda_{n, s}^{\ominus}$ in $\mathcal{M}_{s}(Q)$, then clearly $\lambda_{n, 0}^{\oplus}, \lambda_{n, 0}^{\ominus}, \lambda_{n, s}^{\oplus}, \lambda_{n, s}^{\ominus}$ are nonnegative, $\mu_{n, 0}=f_{n}-\operatorname{div}\left(G_{n}\right)+g_{n}+\lambda_{n, 0}^{\oplus}-\lambda_{n, 0}^{\ominus}$ and $\mu_{n, s}=\lambda_{n, s}^{\oplus}-\lambda_{n, s}^{\ominus}$. In particular we have

$$
0 \leq \mu_{n, s}^{+} \leq \lambda_{n, s}^{\oplus} \quad 0 \leq \mu_{n, s}^{-} \leq \lambda_{n, s}^{\ominus} .
$$

Remark 2.5. Let $\left(\rho_{n}\right)$ be a sequence of $L^{1}(Q)$ functions converging to $\rho$ weakly in $L^{1}(Q)$, let $\left(\sigma_{n}\right)$ be a sequence of functions belonging to $L^{\infty}(Q)$, bounded in the same space and converging a.e. on $Q$ to a function $\sigma$. Then, as a consequence of the Egorov theorem $[\mathrm{Eg}]$, one obtains

$$
\lim _{n \rightarrow \infty} \int_{Q} \sigma_{n} \rho_{n} d x d t=\int_{Q} \sigma \rho d x d t
$$

This property will be often used in Section 4 .

2.6. Renormalized solutions. We are now in position to introduce the notion of renormalized solutions for general measure data.

Definition 2.3. Let $\mu$ be a measure in $\mathcal{M}_{b}(Q), \mu=\mu_{0}+\mu_{s}, \mu_{0} \in \mathcal{M}_{0}(Q), \mu_{s} \in \mathcal{M}_{s}(Q)$. A function $u \in L^{1}(Q)$ is a renormalized solution of problem

$$
\left\{\begin{array}{l}
u_{t}-\operatorname{div}(a(t, x, u, \nabla u))=\mu \text { in } Q:=\Omega \times(0, T), \\
u=0 \text { on }(0, T) \times \partial \Omega, \quad u(0)=u_{0} \text { in } \Omega,
\end{array}\right.
$$

if the following conditions holds

(a) $T_{k}(u) \in L^{p}\left(0, T ; W_{0}^{1, p}(\Omega)\right)$ for every $k>0$;

(b) there exists a sequence of diffuse measures $v^{k} \in \mathcal{M}_{b}(Q)$ such that

$$
v^{k} \rightarrow \mu_{s} \text { tightly as } k \rightarrow+\infty \text {; }
$$

(c) the truncates of $u$ satisfies

$$
\begin{aligned}
& -\int_{Q} T_{k}(u) \varphi_{t} d x d t+\int_{Q} a\left(t, x, T_{k}(u), \nabla T_{k}(u)\right) \cdot \nabla \varphi d x d t \\
& =\int_{Q} \varphi d \mu_{0}+\int_{Q} \varphi d v_{k}+\int_{Q} T_{k}\left(u_{0}\right) \varphi(0) d x
\end{aligned}
$$

for every $k>0$ and $\varphi \in C_{c}^{\infty}([0, T] \times \Omega)$.

Let $W$ be the set of all functions $u \in L^{p}(0, T ; V)$ whose derivative $u_{t} \in L^{p^{\prime}}\left(0, T ; V^{\prime}\right)$ (see (2.4)). Clearly every function $v \in W \cap L^{\infty}(Q)$ with $v(T)=0$ is an admissible test function, so that we can define the renormalized solution $u$ as a solution of

$$
\begin{aligned}
& -\int_{0}^{T}\left\langle T_{k}(u), v_{t}\right\rangle d t+\int_{Q} a\left(t, x, T_{k}(u), \nabla T_{k}(u)\right) \cdot \nabla v d x d t \\
& =\int_{Q} v d \mu_{0}+\int_{Q} v d v_{k}+\int_{\Omega} T_{k}\left(u_{0}\right) v(0) d x .
\end{aligned}
$$

Notice that for every $h \in C^{2}(\mathbb{R})$ such that $h^{\prime}$ and $h^{\prime \prime}$ are bounded and for every $v \in W$ such that $w=h(u)$, the function $\varphi=w \zeta$ is admissible in (c), where $\zeta \in W^{1, \infty}(0, T)$ with compact support in $(0, T)$. The following result is an extension of the result proved in [PPP2, Sect. 4] for diffuse measures

Theorem 2.3. Let $u$ be a renormalized solution according to Definition 2.2, and $\mu$ be split as in (2.7), namely $\mu=$ $f-\operatorname{div}(G)+g_{t}+\mu_{s}^{+}-\mu_{s}^{-}$with $f \in L^{1}(Q),-\operatorname{div}(G) \in L^{p^{\prime}}\left(0, T ; W^{-1, p^{\prime}}(\Omega)\right), g \in L^{p}(0, T ; V)$ and $\left(\mu_{s}^{+}, \mu_{s}^{-}\right)$are the positive and the negative parts of the singular term of the measure $\mu$. Then $u$ satisfies

$$
u-g \in L^{\infty}\left(0, T ; L^{1}(\Omega)\right), \quad T_{k}(u-g) \in L^{p}\left(0, T ; W_{0}^{1, p}(\Omega)\right) \quad \forall k>0 ;
$$




$$
\begin{aligned}
& \lim _{h \rightarrow \infty} \int_{\{h \leq|u-g|<h+1\}} a(t, x, u, \nabla u) \cdot \nabla(u-g) \psi d x d t=\int_{Q} \psi d \mu_{s} \quad \forall \psi \in C_{c}^{\infty}(Q) ; \\
& -\int_{Q} S\left(u_{0}\right) \varphi(0, x) d x-\int_{0}^{T}\left\langle\varphi_{t}, S(u-g)\right\rangle d t+\int_{Q} a(t, x, u, \nabla u) \cdot \nabla\left(S^{\prime}(u-g) \varphi\right) d x d t \\
& =\int_{Q} f S^{\prime}(u-g) \varphi d x d t+\int_{Q} G \cdot \nabla\left(S^{\prime}(u-g) \varphi\right) d x d t
\end{aligned}
$$

for every $S \in W^{2, \infty}(\mathbb{R})$ such that $S^{\prime}$ has compact support on $\mathbb{R}$ and $S(0)=0$ and for every $\varphi \in C_{c}^{\infty}(Q)$.

\section{Some remarks on the truncations}

Our strategy will be to associate to every renormalized solution a sequence of parabolic problems solved by its truncations. If $u$ is a solution in the sense of distributions to problem (1.1) obtained by approximation (in particular if $u$ is a renormalized solution, see [PPP1, Theorem 1.2], then the truncations of $u$ are solutions in the sense of distributions to parabolic problems of the same form with suitable measure data (see [PP, PPP1, PPP2])). The following result gives some details on the form of these measure data. To simplify the proof, we consider just the $p$-Laplacian operator and nonnegative diffuse measures, for more general operators and general measures (see Section 4).

Proposition 3.1. Assume that $\mu \in \mathcal{M}_{b}(Q)$ satisfies the decomposition $\mu=f-\operatorname{div}(G)+g_{t}$ in $\mathcal{D}^{\prime}(Q)$, where $f \in L^{1}(Q)$, $-\operatorname{div}(G) \in L^{p^{\prime}}\left(0, T ; W^{-1, p^{\prime}}(\Omega)\right)$ and $g \in L^{p}\left(0, T ; W_{0}^{1, p}(\Omega)\right)$. If $g \in L^{\infty}(Q)$, then $\mu$ is diffuse.

Proof. See [PPP2, Proposition 3.1].

The following Proposition gives a useful estimate on the solutions introduced in Section 1.

Proposition 3.2. Given $\mu \in \mathcal{M}_{b}(Q) \cap L^{p^{\prime}}\left(0, T ; W^{-1, p^{\prime}}(\Omega)\right)$ and $u_{0} \in L^{2}(\Omega)$, let $u \in L^{p}(0, T ; V)$ be a solution of (1.1). Then,

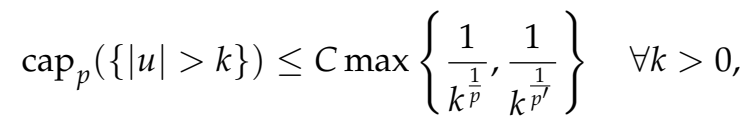

where $C>0$ is a constant depending on $\|\mu\|_{\mathcal{M}_{b}(Q)},\left\|u_{0}\right\|_{L^{1}(Q)}, N, p$ and $\Omega$.

Proof. See [PPP2, Theorem 1.2].

Lemma 3.1. Let $\mu \in \mathcal{M}_{0}(Q)$. Then, for every $\epsilon>0$, there exists $v \in \mathcal{M}_{b}(Q)$ such that

$$
\|\mu-v\|_{\mathcal{M}_{b}(Q)} \leq \epsilon \text { and } v=w_{t}-\Delta_{p} w \text { in } \mathcal{D}^{\prime}(Q),
$$

where $w \in L^{p}\left(0, T ; W_{0}^{1, p}(\Omega)\right) \cap L^{\infty}(Q)$.

Proof. Let $\mu$ be a measure and $\left(\rho_{n}\right)$ a sequence of mollifiers (see Figure 1) satisfying

$$
\rho_{n} \in C_{c}^{\infty}\left(\mathbb{R}^{2 N}\right) \text {, supp } \rho_{n} \subset B_{\frac{1}{n}}(0), \rho_{n} \geq 0 \text { and } \int_{\mathbb{R}^{N+1}} \rho_{n}=1 \text {. }
$$

We define for every $(t, x) \in \mathbb{R} \times \mathbb{R}^{N}$, the measure $\mu_{n}$ as a convolution

$$
\mu_{n}=\left(\rho_{n} * \mu\right)(t, x)=\int_{Q} \rho_{n}(t-s, x-y) d \mu(s, y) .
$$

Now, let us fix $\sigma>0$ and define the function $S_{k, \sigma}: \mathbb{R} \mapsto \mathbb{R}$ by (see Figure 3)

$$
S_{k, \sigma}(s)= \begin{cases}1 & \text { if } s \leq k, \\ 0 & \text { if } s>k+\sigma, \quad T_{k, \sigma}(s)=\int_{0}^{s} S_{k, \sigma}(\eta) d \eta . \\ \text { affine } & \text { elsewhere. }\end{cases}
$$

We denote by $u_{n}$ the solution of (1.1) with datum $\mu_{n}$, we multiply the equation solved by $u_{n}$ by $S_{k, \sigma}\left(u_{n}\right) \varphi$ with 


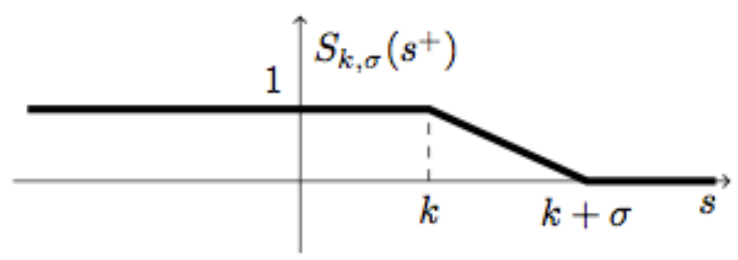

Figure 3. The function $S_{k, \sigma}\left(s^{+}\right)$

$\varphi \in C_{c}^{\infty}(Q)$ to have

$$
\begin{aligned}
& T_{k, \sigma}\left(u_{n}\right)_{t}-\operatorname{div}\left(S_{k, \sigma}\left(u_{n}\right)\left|\nabla u_{n}\right|^{p-2} \nabla u_{n}\right) \\
& \quad=S_{k, \sigma}\left(u_{n}\right) \mu_{n}+\frac{1}{\sigma}\left|\nabla u_{n}\right|^{p} \operatorname{sign}\left(u_{n}\right) \chi_{\left\{k<\left|u_{n}\right| \leq k+\sigma\right\}} \text { in } \mathcal{D}^{\prime}(Q) .
\end{aligned}
$$

Now, we use $\left(1-S_{k, \sigma}\left(u_{n}\right)\right) \operatorname{sign}\left(u_{n}\right)$ as a test function in the same equation to obtain,

$$
\frac{1}{\sigma} \int_{\left\{k \leq\left|u_{n}\right|<k+\sigma\right\}}\left|\nabla u_{n}\right|^{p} \leq \int_{Q}\left(1-S_{k, \sigma}\left(u_{n}\right)\right)\left|\mu_{n}\right| \leq \int_{\left\{\left|u_{n}\right|>k\right\}}\left|\mu_{n}\right| .
$$

Thus, using (3.2) the right-hand side of (3.1) remains bounded in $L^{1}(Q)$ as $\sigma \rightarrow 0$, then we deduce that

$$
T_{k}\left(u_{n}\right)_{t}-\Delta_{p} T_{k}\left(u_{n}\right) \text { is a finite measure in } Q .
$$

Let us set $v_{n}^{k}=T_{k}\left(u_{n}\right)_{t}-\Delta_{p} T_{k}\left(u_{n}\right)$. By (3.1) - (3.2) we have

$$
\begin{aligned}
\int_{Q}\left|v_{n}^{k}\right| d x d t & \leq \liminf _{\sigma \rightarrow 0} \int_{Q}\left|S_{k, \sigma}\left(u_{n}\right) \mu_{n}+\frac{\left|\nabla u_{n}\right|^{p}}{\sigma} \operatorname{sign}\left(u_{n}\right) \chi_{\left\{k \leq\left|u_{n}\right|<k+\sigma\right\}}\right| d x d t \\
& \leq \int_{\left\{\left|u_{n}\right|>k\right\}}\left|\mu_{n}\right| d x d t+\limsup _{\sigma \rightarrow 0} \int_{\left\{k \leq\left|u_{n}\right|<k+\sigma\right\}} \frac{\left|\nabla u_{n}\right|^{p}}{\sigma} d x d t \\
& \leq 2 \int_{\left\{\left|u_{n}\right|>k\right\}}\left|\mu_{n}\right| d x d t .
\end{aligned}
$$

Recall that by Proposition 2.2, the sequence $\left(\mu_{n}\right)$ is equidiffuse. Then by Proposition 3.2 we can fix $k>0$ to obtain the right-hand side of (3.3) smaller than $\epsilon$ for every $n \geq 1$. In particular, $\left(v_{n}^{k}\right)$ remains uniformly bounded in $\mathcal{M}_{b}(Q)$ as $n \rightarrow \infty$. Passing to a subsequence, we may assume that $\left(v_{n}^{k}\right)$ converges weak ${ }^{*}$ in $\mathcal{M}_{b}(Q)$ to some measure $v^{k}$ as $n \rightarrow \infty$. By a classical results of [BDGO], there exists a function $u \in L^{1}(Q)$ such that $u_{n} \rightarrow u$ and $\nabla u_{n} \rightarrow \nabla u$ a.e. in Q. In particular

$$
T_{k}\left(u_{n}\right) \rightarrow T_{k}(u) \text { in } L^{p}\left(0, T ; W_{0}^{1, p}(\Omega)\right) \text { and } \Delta_{p} T_{k}\left(u_{n}\right) \rightarrow \Delta_{p} T_{k}(u) \text { in } L^{p^{\prime}}\left(0, T ; W^{-1, p^{\prime}}(\Omega)\right)
$$

as $n \rightarrow \infty$. Therefore

$$
v^{k}=T_{k}(u)_{t}-\Delta_{p} T_{k}(u)
$$

Since $T_{k}(u) \in L^{p}\left(0, T ; W_{0}^{1, p}(\Omega)\right) \cap L^{\infty}(Q)$. It follows from Proposition 3.1 that $v^{k}$ is a diffuse measure. Moreover, from (3.3) and the lower semi-continuity of the norm with respect to the weak ${ }^{*}$ convergence, we obtain

$$
\left\|v^{k}-\mu\right\|_{\mathcal{M}_{b}(Q)} \leq \liminf _{n \rightarrow \infty} \int_{Q}\left|v_{n}^{k}-\mu_{n}\right| d x d t \leq 2 \liminf _{n \rightarrow \infty} \int_{\left\{\left|u_{n}\right|>k\right\}}\left|\mu_{n}\right| d x d t \leq \epsilon .
$$

This conclude the proof of Lemma 3.1.

The following Lemma, proved in [Pe1], gives a useful estimates on the cut-off functions $\psi_{\delta}$ introduced in [DMOP]. 


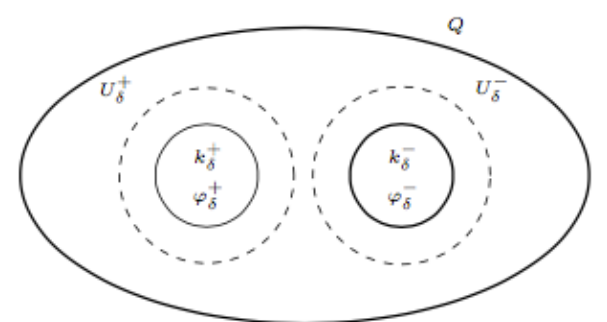

FIGURE 4. The construction of cut-off functions with $K_{\delta}=K_{\delta}^{+} \cup K_{\delta}^{-},\left(K_{\delta}^{+} \cap K_{\delta}^{-}=\emptyset\right)$.

Lemma 3.2. Let $\mu_{s}$ be a nonnegative measure in $\mathcal{M}_{s}(Q)$ concentrated on a subset $E$ of zero $p$-capacity. Then, for every $\delta>0$, there exist a compact set $K_{\delta} \subseteq E$ such that

$$
\mu_{s}\left(E \backslash K_{\delta}\right) \leq \delta
$$

and there exist a function $\psi_{\delta} \in C_{c}^{\infty}(Q)$, such that

$$
\psi_{\delta} \equiv 1 \text { on } K_{\delta}, 0 \leq \psi_{\delta} \leq 1,\left\|\psi_{\delta}\right\|_{S} \leq \delta
$$

Moreover

$$
\int_{Q}\left(1-\psi_{\delta}\right) d \mu \leq \delta
$$

Proof. See [Pe1, Lemma 5].

Remark 3.1. Note that

(i) In [PPP2] it has proved that $u$ is a renormalized solution if and only if for every nonnegative measure $\mu \in$ $\mathcal{M}_{b}(Q) \cap L^{p^{\prime}}\left(0, T ; W^{-1, p^{\prime}}(\Omega)\right)$ and $u_{0} \in L^{2}(\Omega)$, there exist a solution of (1.1) in the usual weak sense. Indeed, in this case $u \in W$ and satisfies the capacitary estimate

$$
\operatorname{cap}_{p}(\{|u|>k\}) \leq C \max \left\{\frac{1}{k^{\frac{1}{p}}}, \frac{1}{k^{\frac{1}{p^{\prime}}}}\right\} \quad \forall k \geq 1,
$$

where $C>0$ is a constant depending on $\|\mu\|_{\mathcal{M}_{b}(Q)},\left\|u_{0}\right\|_{L^{1}(\Omega)}$ and $p$.

(ii) In [PPP2, Lemma 2.1], it is shown that since $u \in W$, the solution $u$ is identified with its cap $p_{p}$-quasi-continuous representative, obtaining the well-defined quantity cap $p(\{|u|>k\})$.

\section{The stability result}

Theorem 4.1. Assume that $a(t, x, s, \zeta)$ satisfies (2.1) - (2.3). Fixed a Radon measure $\mu$ such that $\mu=f-\operatorname{div}(G)+g_{t}+$ $\mu_{s}^{+}-\mu_{s}^{-}$; let $\left(\mu_{n}\right), n \in \mathbb{N}$, be a sequence of Radon measures having a splitting $\left(f_{n}, \operatorname{div}\left(G_{n}\right), g_{n}, \lambda_{n}^{\oplus}, \lambda_{n}^{\ominus}\right)$ converging to $\mu$ as $n$ tends to $+\infty$. Let $u_{n}$ be a renormalized solution to the problem

$$
\left\{\begin{array}{l}
u_{t}^{n}-\operatorname{div}\left(a\left(t, x, u_{n}, \nabla u_{n}\right)\right)=\mu_{n} \text { in } Q:=\Omega \times(0, T), \\
u_{n}=0 \text { on }(0, T) \times \partial \Omega, \quad u_{n}(0)=u_{0}^{n} \text { in } \Omega .
\end{array}\right.
$$

Then there exists a subsequence of $\left(u_{n}\right)$ (resp. $\left(u_{n}-g_{n}\right)$ ), still denoted by $u_{n}$ (resp. $\left(u_{n}-g_{n}\right)$ ), which converges almost everywhere to a renormalized solution of (1.1) with datum $\mu$. Moreover, for every $k \in \mathbb{R}^{+}$the truncations $\left(T_{k}\left(u_{n}\right)\right)($ resp. $\left(T_{k}\left(u_{n}-g_{n}\right)\right)$ ) converges to $T_{k}(u)\left(r e s p . T_{k}(u-g)\right)$ in the weak topology of $L^{p}\left(0, T ; W_{0}^{1, p}(\Omega)\right)$, and the gradient $\left(\nabla u_{n}\right)$ (resp. $\left.\nabla\left(u_{n}-g_{n}\right)\right)$ converges to $\nabla u($ resp. $\nabla(u-g))$ a.e. in $Q$. 
Proof. Let $u_{n}$ be a renormalized solution to (4.1), that is $T_{k}\left(u_{n}\right) \in L^{p}\left(0, T ; W_{0}^{1, p}(\Omega)\right)$ and satisfies

$$
\begin{aligned}
\int_{0}^{T} & \left\langle\left(u_{n}-g_{n}\right)_{t}, \varphi\right\rangle d t+\int_{Q} a\left(t, x, u_{n}, \nabla u_{n}\right) \cdot \nabla \varphi d x d t \\
& =\int_{Q} f_{n} \varphi d x d t-\int_{0}^{T}\langle\operatorname{div}(G), \varphi\rangle d x d t+\int_{Q} \varphi d\left(\lambda_{n}^{\oplus}-\lambda_{n}^{\ominus}\right)
\end{aligned}
$$

for every $\varphi \in L^{p}\left(0, T ; W_{0}^{1, p}(\Omega)\right) \cap L^{\infty}(Q), \varphi_{t} \in L^{p^{\prime}}\left(0, T ; W^{-1, p^{\prime}}(\Omega)\right)$, with $\varphi(T, 0)=0$.

As in [Pe1, Sect. 6], we can prove that there exist a subsequences (still denoted by $\left.\left(u_{n}\right)\right)\left(\right.$ resp. $\left.\left(u_{n}-g_{n}\right)\right)$ and a measurable function $u$ (resp. $u-g$ ), which is finite almost everywhere in $Q$, such that the following properties holds:

(i) $\left(u_{n}\right)$ (resp. $\left.\left(u_{n}-g_{n}\right)\right)$ converges to $u$ (resp. $u-g$ ) a.e. in $Q$;

(ii) For every $k \in \mathbb{R}^{+}$, the sequence $\left(T_{k}\left(u_{n}\right)\right)$ (resp. $\left(T_{k}\left(u_{n}-g_{n}\right)\right)$ ) converges to $T_{k}(u)$ (resp. $\left.T_{k}(u-g)\right)$ in the weak topology of $L^{p}\left(0, T ; W_{0}^{1, p}(\Omega)\right)$;

(iii) $\left(\nabla u_{n}\right)$ (resp. $\left.\left(\nabla\left(u_{n}-g_{n}\right)\right)\right)$ converges to $\nabla u$ (resp. $\left.\nabla(u-g)\right)$ a.e. in $Q$;

(iv) $a\left(t, x, u_{n}, \nabla u_{n}\right)$ converges to $a(t, x, u, \nabla u)$ in the strong topology of $L^{q}\left(0, T ; W_{0}^{1, q}(\Omega)\right)$ for every $q<p-$ $\frac{N}{N+1}$, while $a\left(t, x, T_{k}\left(u_{n}\right), \nabla T_{k}\left(u_{n}\right)\right)$ converges to $a\left(t, x, T_{k}(u), \nabla T_{k}(u)\right)$ in the weak topology of $\left(L^{p^{\prime}}(Q)\right)^{N}$ for every $k \in \mathbb{R}^{+}$.

In the flow of the arguments in [Pe1], the next step involves a long and technical proof of the strong convergence of $\left(T_{k}\left(u_{n}-g_{n}\right)\right)$ to $T_{k}(u-g)$ in $L^{p}\left(0, T ; W_{0}^{1, p}(\Omega)\right)$, which is needed in order to pass to the limit in the equations solved by $u_{n}$ and to obtain that $u$ is a renormalized solution of (2.12). As a consequence of Theorem 4.1, we obtain the following result

Theorem 4.2. Let $\left(u_{n}\right)$ (resp. $\left.\left(u_{n}-g_{n}\right)\right)$ and $u$ (resp. $u-g$ ) be the functions defined in Theorem 4.1. Then for every $k \in$ $\mathbb{R}^{+}$, the sequence $T_{k}(u)\left(\right.$ resp. $T_{k}(u-g)$ ) converges to $T_{k}(u)\left(\right.$ resp. $\left.T_{k}(u-g)\right)$ in the strong topology of $L^{p}\left(0, T ; W_{0}^{1, p}(\Omega)\right.$ ).

Proof. See [Pe1, Theorem 5].

We now propose a different passage to the limit that uses the equations solved by $\left(T_{k}\left(u_{n}\right)\right)$ and that does not need the strong convergence of the truncations. While the same problem was studied in [PP] for nonnegative data, here we want to generalize this result to changing sign measure data.

Proof of Theorem 4.1 (continued) : Step 1. Estimates in $L^{1}(Q)$. By Theorem 2.2, there exists two measures $\mu_{0} \in \mathcal{M}_{0}(Q)$ and $\mu_{s} \in \mathcal{M}_{s}(Q)$ concentrated on a set of zero $p$-capacity such that $\mu_{n}=\rho_{n} * \mu=\mu_{0}^{n}+\mu_{s}^{n}$ with $\mu_{0}^{n}=\rho_{n} * \mu_{0}$ and $\mu_{s}^{n}=\rho_{n} * \mu_{s}$ such that the truncates $T_{k}\left(u_{n}\right)$ satisfies

$$
\int_{0}^{T}\left\langle\left(T_{k}\left(u_{n}\right)\right)_{t}, v\right\rangle d t+\int_{Q} a\left(t, x, T_{k}\left(u_{n}\right), \nabla T_{k}\left(u_{n}\right)\right) \cdot \nabla v d x d t=\int_{0}^{T}\left\langle\mu_{n}, v\right\rangle d t \quad \forall v \in W,
$$

where $\langle\cdot, \cdot\rangle$ denotes the duality between $V$ and $V^{\prime}$. On the other hand, if we consider the function $h_{k, \sigma}(s): \mathbb{R} \mapsto \mathbb{R}$ defined by (see Figure 5)

$$
h_{k, \sigma}(s)= \begin{cases}0 & \text { if }|s| \leq k \\ 1 & \text { if }|s|>k+\sigma, \quad H_{k, \sigma}(s)=\int_{0}^{s} h_{k, \sigma}(\eta) d \eta . \\ \text { affine } & \text { elsewhere }\end{cases}
$$

Choosing $v=h_{k, \sigma}\left(u_{n}\right) \operatorname{sign}\left(u_{n}\right)$ as test function in (4.1), we obtain 


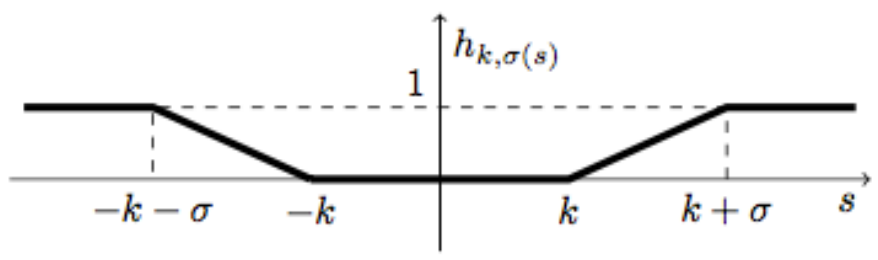

FIGURE 5. The function $h_{k, \sigma}(s)$

$$
\begin{aligned}
& \frac{1}{\sigma} \int_{Q} a\left(t, x, u_{n}, \nabla u_{n}\right) \cdot \nabla u_{n} \operatorname{sign}\left(u_{n}\right) \chi_{\left\{k<\left|u_{n}\right|<k+\sigma\right\}} d x d t \\
& =\int_{Q} h_{k, \sigma}\left(u_{n}\right) d \mu_{n}+\int_{\Omega} H_{k, \sigma}\left(u_{0}^{n}\right)-\int_{\Omega} H_{k, \sigma}\left(u_{n}(T)\right) \\
& \quad \leq \int_{\left\{\left|u_{n}\right|>k\right\}} d \mu_{n}+\int_{\left\{\left|u_{0}^{n}\right|>k\right\}} d x
\end{aligned}
$$

which easily implies the estimate in $L^{1}(Q)$ on the energy term

$$
\frac{1}{\sigma} \int_{\left\{k<\left|u_{n}\right| \leq k+\sigma\right\}} a\left(t, x, u_{n}, \nabla u_{n}\right) \cdot \nabla u_{n} \operatorname{sign}\left(u_{n}\right) d x d t \leq C,
$$

for every $n \geq 1$, then there exist a bounded measure $\lambda_{k}^{n}$ such that, as $\sigma$ goes to zero

$$
\frac{1}{\sigma} a\left(t, x, u_{n}, \nabla u_{n}\right) \cdot \nabla u_{n} \chi_{\left\{k<\left|u_{n}\right|<k+\sigma\right\}} \rightarrow \lambda_{k}^{n} \text { weakly* in } \mathcal{M}_{b}(Q) .
$$

Step 2. Equations for the truncations. To prove $(c)$, we consider the primitive function $T_{k, \sigma}(s)$ and $\varphi \in C_{c}^{\infty}(Q)$, notice that $T_{k, \sigma}(s)$ converges pointwise to $T_{k}(s)$ as $\sigma$ goes to zero. We multiply by $T_{k, \sigma}^{\prime}\left(u_{n}\right) \varphi$ in (4.1) to obtain

$$
T_{k, \sigma}\left(u_{n}\right)_{t}-\operatorname{div}\left(S_{k, \sigma}\left(u_{n}\right) a\left(t, x, u_{n}, \nabla u_{n}\right)\right)=S_{k, \sigma}\left(u_{n}\right) \mu_{n}+\frac{1}{\sigma} a\left(t, x, u_{n}, \nabla u_{n}\right) \cdot \nabla u_{n} \operatorname{sign}\left(u_{n}\right) \chi_{\left\{k<\left|u_{n}\right|<k+\sigma\right\}} \text { in } \mathcal{D}^{\prime}(Q)
$$

which yields as $\sigma$ goes to 0

$$
T_{k}\left(u_{n}\right)_{t}-\operatorname{div}\left(a\left(t, x, u_{n}, \nabla u_{n} \chi_{\left\{\left|u_{n}\right| \leq k\right\}}\right)\right)=\left(\mu_{0}^{n}+\mu_{s}^{n}\right) \chi_{\left\{\left|u_{n}\right| \leq k\right\}}+\lambda_{n}^{k} \operatorname{in} \mathcal{D}^{\prime}(Q)
$$

using $\mu_{0}^{n}=\mu_{0}^{n} \chi_{\left\{\left|u_{n}\right| \leq k\right\}}+\mu_{0}^{n} \chi_{\left\{\left|u_{n}\right| \geq k\right\}}$, we obtain

$$
T_{k}\left(u_{n}\right)_{t}-\operatorname{div}\left(a\left(t, x, T_{k}\left(u_{n}\right), \nabla T_{k}\left(u_{n}\right)\right)\right)-\mu_{0}^{n}=\mu_{s}^{n} \chi_{\left\{\left|u_{n}\right| \leq k\right\}}-\mu_{0}^{n} \chi_{\left\{\left|u_{n}\right| \geq k\right\}}+\lambda_{n}^{k} \text { in } \mathcal{D}^{\prime}(Q) .
$$

Let us set

$$
v_{n}^{k}=\mu_{s}^{n} \chi_{\left\{\left|u_{n}\right|<k\right\}}-\mu_{0}^{n} \chi_{\left\{\left|u_{n}\right| \geq k\right\}}+\lambda_{n}^{k}
$$

from the fact that $\mu_{0}^{n}$ and $\mu_{s}^{n}$ are equidiffuse measures, we have that there exist a constant $\tilde{M}>0$ such that $\mu_{s}^{n}+\mu_{0}^{n} \leq \tilde{M}$ for every $n \in \mathbb{N}^{*}$. Then for every $k \in \mathbb{R}^{+}$there exist $C>0$ (possibly depending on $k$ ) such that

$$
\left\|\lambda_{k}^{n}\right\|_{L^{1}(Q)} \leq C, \quad\left\|v_{n}^{k}\right\|_{L^{1}(Q)} \leq C,
$$

and hence there exist $v^{k} \in \mathcal{M}(Q)$ which is the weak ${ }^{*}$ limit in $\mathcal{M}_{b}(Q)$ of the sequence $v_{n}^{k}$ since $\chi_{\left\{\left|u_{n}\right|<k\right\}}$ converges to $\chi_{\{|u|<k\}}$ a.e. in $Q$ and weakly* in $L^{\infty}(Q)$ for every $k \in \mathbb{R}^{+}$, Then from convergences $(i)-(i v)$, we obtain

$$
T_{k}(u)_{t}-\operatorname{div}\left(a\left(t, x, T_{k}(u), \nabla T_{k}(u)\right)\right)=\mu_{0}+v^{k} \text { in } \mathcal{D}^{\prime}(Q) .
$$

Let us consider the next identity obtained by subtraction

$$
\begin{aligned}
& -\int_{Q} u_{n} \varphi_{t} d x d t+\int_{Q} a\left(t, x, u_{n}, \nabla u_{n}\right) \cdot \nabla \varphi d x d t-\int_{Q} \varphi d \mu_{0}^{n}-\int_{Q} \varphi d \mu_{s}^{n}-\int_{\Omega} \varphi(0) u_{0}^{n} d x \\
& =-\int_{Q} T_{k}(u) \varphi_{t} d x d t+\int_{Q} a\left(t, x, T_{k}(u), \nabla T_{k}(u)\right) \cdot \nabla \varphi d x d t-\int_{Q} \varphi d \mu_{0}-\int_{Q} \varphi d v^{k}-\int_{\Omega} \varphi(0) T_{k}\left(u_{0}\right) d x
\end{aligned}
$$


for all $\varphi \in C_{c}^{\infty}(Q)$. We can pass to the limit in $n$, using the basic compactness convergences $(i)-(i v)$ to get

$$
\lim _{n \rightarrow \infty} \lim _{k \rightarrow \infty} \int_{Q} \varphi d\left(\mu_{s}^{n}-v^{k}\right) d x d t=0
$$

for every $\varphi \in C_{c}^{\infty}(Q)$, that is

$$
v^{k}=\mu_{s}+\omega(n, k) \text { in } \mathcal{D}^{\prime}(Q) .
$$

Fixed $k \in \mathbb{R}^{+}$, let us now consider the sequence $\psi_{\delta}$ of cut-off functions in $C_{c}^{\infty}(Q)$ such that $0 \leq \psi_{\delta} \leq 1$ and $S$-converging to the null function as $\delta \rightarrow 0$, we put $\psi_{\delta} \varphi$ with $\varphi \in C_{c}^{1}(\bar{Q})$ as test function in (4.4) to obtain

$$
\begin{aligned}
\int_{Q} \psi_{\delta} \varphi d v^{k} & =\int_{E} \psi_{\delta} \varphi d \mu_{s}+\omega(k) \\
& =\int_{K_{\delta}} \varphi d \mu_{s}+\int_{E \backslash K_{\delta}} \psi_{\delta} \varphi d \mu_{s}+\omega(k) . \\
& \leq \int_{Q} \varphi d \mu_{s}+\delta\|\varphi\|_{L^{\infty}(Q)}+\omega(k) .
\end{aligned}
$$

So that, passing to the limit in $\delta$,

$$
\int_{Q} \psi_{\delta} \varphi d v^{k}=\int_{Q} \varphi d \mu_{s}+\omega(k, \delta)
$$

Step 3. The limit of $v^{k}$. Let us now consider the measures $\mu_{0}^{n}$ and $\mu_{s}^{n}$. Choosing as test function in (4.1) the function $h_{k, \sigma}\left(u_{n}\right)\left(1-\psi_{\delta}\right)$, where $\psi_{\delta} \in C_{c}^{\infty}(Q)$ and $h_{k, \sigma}$ is defined in Figure 5, we obtain

$$
\begin{aligned}
& \frac{1}{\sigma} \int_{\left\{k<\left|u_{n}\right|<k+\sigma\right\}} a\left(t, x, u_{n}, \nabla u_{n}\right) \cdot \nabla u_{n}\left(1-\psi_{\delta}\right) d x d t-\int_{Q} a\left(t, x, u_{n}, \nabla u_{n}\right) \cdot \nabla \psi_{\delta} h_{k, \sigma}\left(u_{n}\right) d x d t \\
& =\int_{Q} h_{k, \sigma}\left(u_{n}\right)\left(1-\psi_{\delta}\right) d\left(\mu_{0}^{n}+\mu_{s}^{n}\right)-\int_{Q} H_{k, \sigma}\left(u_{n}(t, x)\right)\left(\psi_{\delta}\right)_{t} d x d t+\int_{\Omega} H_{k, \sigma}\left(u_{0}^{n}(x)\right)\left(1-\psi_{\delta}(0)\right) d x .
\end{aligned}
$$

Passing to the limit as $\delta$ goes to 0 and recall that $u_{n}$ (resp. $a\left(t, x, u_{n}, \nabla u_{n}\right)$ ) converges strongly to $u$ (resp. $a(t, x, u, \nabla u))$ in $L^{1}(Q)$, we obtain

$$
\int_{Q} H_{k, \sigma}\left(u_{n}(t, x)\right)\left(\varphi_{\delta}\right)_{t} d x d t=\omega(k) \text { and } \int_{Q} a\left(t, x, u_{n}, \nabla u_{n}\right) \cdot \nabla \psi_{\delta} h_{k, \sigma}\left(u_{n}\right) d x d t=\omega(n, k),
$$

for fixed $\delta>0$ and by the equi-diffuse property of the measure $\mu_{0}^{n}$ we get

$$
\left|\int_{Q} \mu_{0}^{n} h_{k, \sigma}\left(u_{n}\right)\left(1-\psi_{\delta}\right) d x d t\right| \leq \int_{\left\{\left|u_{n}\right|>k\right\}} \mu_{0}^{n}\left(1-\psi_{\delta}\right) d x d t=\omega(n, k) .
$$

On the other hand, Lemma 3.2 implies that

$$
\left|\int_{Q} \mu_{s}^{n} h_{k, \sigma}\left(u_{n}\right)\left(1-\psi_{\delta}\right) d x d t\right| \leq \int_{Q} \mu_{s}^{n}\left(1-\psi_{\delta}\right) d x d t=\omega(n, k)
$$

and hence

$$
\frac{1}{\sigma} \int_{\left\{k<\left|u_{n}\right| \leq k+\sigma\right\}} a\left(t, x, u_{n}, \nabla u_{n}\right) \cdot \nabla u_{n} \varphi\left(1-\psi_{\delta}\right)=\omega(\sigma, n, k, \delta) .
$$

Choosing $\left(1-\psi_{\delta}\right) \varphi$ as test function in (4.1), we have

$$
\begin{aligned}
\int_{Q} \varphi\left(1-\psi_{\delta}\right) d v_{n}^{k}= & \int_{Q} \varphi\left(1-\psi_{\delta}\right) d \lambda_{n}^{k}+\int_{\left\{\left|u_{n}\right| \leq k\right\}} \varphi\left(1-\psi_{\delta}\right) d \mu_{s}^{n}-\int_{\left\{\left|u_{n}\right|>k\right\}} \varphi\left(1-\psi_{\delta}\right) d \mu_{0}^{n} \\
= & \lim _{\sigma \rightarrow 0} \frac{1}{\sigma} \int_{\left\{k<\left|u_{n}\right| \leq k+\sigma\right\}} a\left(t, x, u_{n}, \nabla u_{n}\right) \cdot \nabla u_{n} \varphi\left(1-\psi_{\delta}\right) d x d t \\
& +\int_{\left\{\left|u_{n}\right| \leq k\right\}} \varphi\left(1-\psi_{\delta}\right) d \mu_{s}^{n}-\int_{\left\{\left|u_{n}\right|>k\right\}} \varphi\left(1-\psi_{\delta}\right) d \mu_{0}^{n} \\
= & \omega(\sigma, n, k)
\end{aligned}
$$


which easily implies that there exists $v^{k} \in \mathcal{M}(Q)$ such that

$$
\int_{Q} \varphi\left(1-\psi_{\delta}\right) d v_{n}^{k}=\omega(k, \delta)
$$

for almost every $k \in \mathbb{R}^{+}$. Hence we can apply an easy density argument in $\varphi \in C(\bar{Q})$, obtaining that for every $\varphi \in C^{\infty}(\bar{Q})$, there exists a sequence of cut-off functions such that

$$
\int_{Q} \varphi d v^{k}=\int_{Q} \varphi \psi_{\delta} d v^{k}+\int_{Q} \varphi\left(1-\psi_{\delta}\right) d v^{k}
$$

this implies that the sequence of measures $v^{k}$ converges in the narrow topology of measures to a measure $\mu_{s}$. In addition, the singular part $\mu_{s}$ of $\mu$ can be estimated by

$$
\lim _{h \rightarrow \infty} \int_{\{h<|u| \leq h+1\}} a(t, x, u, \nabla u) \cdot \nabla u \varphi d x d t=\int_{Q} \varphi d \mu_{s}
$$

for every $\varphi \in C_{c}^{\infty}(Q)$ and hence, the measure $\mu_{s}$ is concentrated by the energy of the approximating solutions.

\section{Equation with absorption}

In this part, we shall continue the study of the stability of renormalized solutions to nonlinear parabolic equations with measures. But from a different point of view, here we investigate the existence and uniqueness of the following nonlinear initial boundary value problems with absorption term and diffuse measure data

$$
\begin{cases}b(u)_{t}-\operatorname{div}(a(t, x, u, \nabla u))+h(u)=\mu & \text { in } Q, \\ u=0 & \text { on }(0, T) \times \partial \Omega, \\ b(u)=b\left(u_{0}\right) & \text { in }(0, T) \times \Omega,\end{cases}
$$

where $p>2-\frac{1}{N+1}, \mu$ is a Radon measure which is diffuse on $Q\left(\mu_{s}=0\right), b$ is a $C^{1}$-increasing function which satisfies $0<b_{0} \leq b^{\prime}(s) \leq b_{1}$ (for positive constants $b_{0}$ and $\left.b_{1}\right)$ such that $b\left(u_{0}\right)$ is an element of $L^{1}(\Omega)$ and $h: \mathbb{R} \mapsto \mathbb{R}$ is a continuous function such that $h(s) s \geq 0$ for every $|s| \geq L$ with $L \geq 0$ (odd functions for example). Equations (5.1) could be viewed as a generalization of the usual equations (1.1). It's a rather typical nonlinear problems (called standard porous medium models) with lower order term. Problems of this kind are interesting from the purely mathematical point of view (see [V]). Besides, they have potential applications in various fields such as continuous mechanics [L2], population dynamics [BM] and image processing [GSZW], etc. Perhaps for these reasons, such a field has attracted more and more attention and has a development in recent years, see [BPR, AA2, AA3] and the large amounts of references therein. Note that the interest of problems as (5.1) (with $b=1$ and smooth data) is studied in [BCW] in connection with the study of conservation laws and in the theory of non-Newtonian fluids. A complete setting of the theory, in the parabolic case with smooth data, was developed over the years starting by seminal papers [BR1, BPR], until the remarkable improvements given in [BF] $\left(\mu \in L^{1}\left(0, T ; L^{p}(\Omega)\right)\right.$ and $\left.h=0\right)$. Again in the parabolic case where $h(s)=-\operatorname{div}(\Phi(t, x, s))$ is a lower order term with $\Phi$ induces a lack of coercivity was settled in [BRM] (see also [ABR] for $(x, u)$-dependence of the function $b$ ). The case of possibly diffuse measure as data in (5.1) is, by far, are limited. If $\mu_{s}=0$ and $b(u)=b(x, u)$ we refer to [MR1] (see also [BRM, MR2, AA3]) for a complete account on generalized porous medium equations in the context of renormalized solutions. Moreover the case of unbounded nonlinearities has been treated in [BGR, BR2] and parabolic equations with natural growth terms have been obtained in [AR] (see also [BP1] and references therein for primary results). Finally, concerning the Stefan model case, for suitably smooth data $f$ and $\mu$, the existence of solutions to problems as in (5.1) was investigated in [BP2]. In order to deal with nonlinear equations where the source term is a diffuse measure; the authors in [BPR] introduced a renormalized formulation that is based on the representation (2.6). However, in context with the elliptic case, such representation, as well as the formulation suggested in [DPP], is not suitable to handle the case of absorption terms as in (5.1). The main reason is that a solution of

$$
b(u)_{t}-\operatorname{div}(a(t, x, u, \nabla u))+h(u)=f-\operatorname{div}(G)+g_{t} \text { in } Q
$$


is meant in the sense that $v=b(u)-g$ satisfies

$$
v_{t}-\operatorname{div}\left(a\left(t, x, b^{-1}(v+g), \nabla b^{-1}(v+g)\right)\right)+h\left(b^{-1}(v+g)\right)=f-\operatorname{div}(G) \text { in } Q .
$$

The same approach for problem (1.1) would transform the absorption term $h(u)$ into $h\left(b^{-1}(v+g)\right)$. However, since no growth restriction is made on $h$, this term can be easily handled if $g$ is not bounded.

In this part, we overcome this obstruction by developing the approach of Section 4 to deal with absorption term. In this way, we establish new properties of renormalized solutions (related to different type of definitions) and new results for parabolic equations (5.1). The next definition in certainly closer to the one used in [AA2] and to the one of the existing formulations in Section 4.

Definition 5.1. Let $\mu \in \mathcal{M}_{b}(Q)$. A function $u \in L^{1}(Q)$ is a renormalized solution of problem (5.1) if $T_{k}(b(u)) \in$ $L^{p}\left(0, T ; W_{0}^{1, p}(\Omega)\right)$ for every $k>0, h(u) \in L^{1}(Q)$, and if there exist a sequence $\left(\lambda_{k}\right)$ in $\mathcal{M}_{0}(Q)$ such that

$$
\lim _{k \rightarrow \infty}\left\|\lambda_{k}\right\|_{\mathcal{M}_{0}(Q)}=0,
$$

and

$$
\begin{aligned}
& -\int_{Q} T_{k}(b(u)) \varphi_{t} d x d t+\int_{Q} a\left(t, x, T_{k}(u), \nabla T_{k}(u) \cdot \nabla \varphi d x d t+\int_{Q} h(u) \varphi d x d t\right. \\
& =\int_{Q} \varphi d \mu+\int_{Q} \varphi d \lambda_{k}+\int_{\Omega} T_{k}\left(u_{0}\right) \varphi(0, x) d x
\end{aligned}
$$

for every $k>0$ and $\varphi \in C_{c}^{\infty}([0, T] \times \Omega)$.

Now, recall the definition of distributional solution of (5.1) and notice that such a definition makes sense for any measure $\mu$, not necessarily diffuse, even if in our context we are always dealing with diffuse and singular measures.

Definition 5.2. A function $u \in L^{1}(Q)$ is a distributional solution of problem (5.1) if $T_{k}(b(u)) \in L^{p}\left(0, T ; W_{0}^{1, p}(\Omega)\right)$ for every $k>0,|\nabla u|^{p-1} \in L^{1}(Q), h(u) \in L^{1}(Q)$ and if

$$
-\int_{Q} b(u) \varphi_{t} d x d t+\int_{Q} a(t, x, u, \nabla u) \cdot \nabla \varphi d x d t+\int_{Q} h(u) \varphi d x d t=\int_{Q} \varphi d \mu+\int_{\Omega} b\left(u_{0}\right) \varphi(0, x) d x
$$

for any $\varphi \in C_{c}^{\infty}([0, T] \times \Omega)$.

We then have

Proposition 5.1. If $u$ is a renormalized solution of (5.1), then for every $k>0$ and $\tau \leq T$,

$$
\int_{\Omega} \Theta_{k}(\tau) d x+\int_{0}^{\tau} \int_{\Omega} b^{\prime}(u)\left|\nabla T_{k}(b(u))\right|^{p} d x d t+\int_{0}^{\tau} \int_{\Omega} h(u) u d x d t \leq C k\left(\|\mu\|_{\mathcal{M}(Q)}+\left\|b\left(u_{0}\right)\right\|_{L^{1}(\Omega)}\right),
$$

where $\Theta_{k}(s)=\int_{0}^{s} T_{k}(t) d t$. Therefore, $b(u) \in L^{\infty}\left(0, T ; L^{1}(\Omega)\right),|\nabla u|^{p-1}$ and $a(t, x, u, \nabla u)$ are in $L^{r}(Q)$ for any $r<\frac{N+p^{\prime}}{N+1}$. Moreover, $u$ is a distributional solution.

We obtain in this way a new approach to solve standard porous medium problems involving measures

Theorem 5.1. Let $\mu \in \mathcal{M}_{0}(Q)$ and $b\left(u_{0}\right) \in L^{1}(\Omega)$. Let $h: \mathbb{R} \mapsto \mathbb{R}$ be a continuous function satisfying

$$
h(s) s \geq 0 \text { for every }|s|>L
$$

for some $L \geq 0$. Then, (5.1) admits a renormalized solution (which is, in particular, a distributional solution). If in addition $h$ is nondecreasing, then the renormalized solution is unique.

Proof. The proof of Theorem 5.1 strongly relies on an estimate developed in [AA3, Proposition 5.2].

Proposition 5.2. Given $\mu \in \mathcal{M}_{0}(Q) \cap L^{p^{\prime}}\left(0, T ; W^{-1, p^{\prime}}(\Omega)\right)$ and $b\left(u_{0}\right) \in L^{1}(\Omega)$, let $u \in W$ be the weak solution of $(5.1)$. Then

$$
\operatorname{cap}_{p}(\{|b(u)|>k\}) \leq C \max \left\{\frac{1}{k^{\frac{1}{p}}}, \frac{1}{k^{\frac{1}{p^{\prime}}}}\right\} \quad \forall k \geq 1,
$$

where $C>0$ is a constant depending on $\|\mu\|_{\mathcal{M}_{0}(Q)},\left\|b\left(u_{0}\right)\right\|_{L^{1}(\Omega)},\left\|b_{0}\right\|_{L^{p^{\prime}}(Q)}, \alpha, \beta, p$ and $\Omega$. 
Proof. See [AA2, Proposition 2.2].

Now, the existence of solution is obtained as limit of solutions $u_{n}$ corresponding to a smooth approximation $\mu_{n}$ of the measure $\mu$. In this procedure, the difficult point is to prove the $L^{1}$-convergence of the lower order term $h\left(u_{n}\right)$. When the sequence $\left(\mu_{n}\right)$ is strongly (or even weakly) converging in $L^{1}(Q)$, this is usually deduced (see [GM]) from the estimate

$$
\int_{\left\{\left|b\left(u_{n}\right)\right|>k\right\}}\left|h\left(u_{n}\right)\right| \leq \int_{\left\{\left|b\left(u_{n}\right)\right|>k\right\}}\left|\mu_{n}\right|
$$

using the equi-integrability of $\left(\mu_{n}\right)$. In our case, we extend this idea in the following sense: the level sets $\left\{\left|b\left(u_{n}\right)\right|>k\right\}$ are proved to have uniformly small capacity (Proposition 5.2) and the sequence $\left(\mu_{n}\right)$ is chosen to be equi-diffuse (Definition 2.1) (coupling the capacitary estimate with the equi-diffuse property of $\left(\mu_{n}\right)$, the righthand side of (5.2) is uniformly small, implying the $L^{1}$ - convergence of $\left.\left(h\left(u_{n}\right)\right)\right)$. Finally, when $h$ is nondecreasing, we obtain uniqueness by proving that the $L^{1}$-contraction property holds for renormalized solutions.

5.1. Approximation scheme and a priori estimates. In order to prove the existence of a renormalized solution, we consider the convolution $\mu_{n}=\left(\rho_{n} * \mu\right)$ introduced in Lemma 3.1. In this way, we use the equi-diffusion property (Proposition 2.1) and we will state basic a priori estimates on the approximating solutions and the convergence results in a slightly more general form which can be applied in case of lower-order terms. Consider the following scheme of approximation

$$
\begin{cases}\left(b\left(u_{n}\right)\right)_{t}-\operatorname{div}\left(a\left(t, x, u_{n}, \nabla u_{n}\right)\right)+h\left(u_{n}\right)=\mu_{n} & \text { in } Q, \\ b\left(u_{n}\right)=b\left(u_{0}^{n}\right) & \text { on }\{0\} \times \Omega, \\ u_{n}=0 & \text { in }(0, T) \times \partial \Omega .\end{cases}
$$

where $b\left(u_{0}^{n}\right)$ is a sequence converging strongly to $b\left(u_{0}\right)$ in $L^{1}(Q)$ and $h_{n}\left(u_{n}\right)$ is a sequence of smooth functions, bounded in $L^{1}(Q)$, that converges strongly in $L^{1}(Q)$ to $h(u)$. The existence of such approximation is obtained by standard convolution arguments stated in Sections 2,4. As usual for nonlinear operators with measure data, we will need the following proposition that collects some known results in the literature.

Proposition 5.3. Let $\mu \in \mathcal{M}_{0}(Q), b\left(u_{0}^{n}\right) \in L^{1}(\Omega)$, with $\sup \left|\mu_{n}\right|(Q)<\infty$ and $\left\|b\left(u_{0}^{n}\right)\right\|_{L^{1}(\Omega)}<\infty$. Let $\left(u_{n}\right)$ be a sequence of renormalized solutions of (5.3). Then there exists $C>0$ such that

$$
\left\|u_{n}\right\|_{L^{\infty}\left(0, T ; L^{1}(\Omega)\right)} \leq C, \quad \int_{Q}\left|\nabla T_{k}\left(u_{n}\right)\right|^{p} d x d t \leq C k
$$

for every $n$ and for every $k>0$. Moreover there exists a subsequence, still denoted by $\left(u_{n}\right)$ and a measurable function $u$ such that the following convergence hold

(i) $u_{n}$ (res. $\left.b\left(u_{n}\right)\right)$ converges to $u($ resp. $b(u))$ a.e. in $Q$;

(ii) $u, b(u)$ belongs to $L^{\infty}\left(0, T ; L^{1}(\Omega)\right)$ and for every $k>0$, the sequence $\left(T_{k}\left(b\left(u_{n}\right)\right)\right)$ converges to $T_{k}(b(u)) \in$ $L^{p}\left(0, T ; W_{0}^{1, p}(\Omega)\right)$ in the weak topology of $L^{p}\left(0, T ; W_{0}^{1, p}(\Omega)\right)$;

(iii) $\nabla u_{n}$ converges to $\nabla u$ a.e. in $Q$;

(iv) $a\left(t, x, u_{n}, \nabla u_{n}\right)$ converges to $a(t, x, u, \nabla u)$ in the strong topology of the space $L^{q}\left(0, T ; W_{0}^{1, q}(\Omega)\right)$ for every $q<$ $p-\frac{N}{N+1}$

(iv) $\left|\nabla u_{n}\right|^{p-2} \nabla u_{n}$ converges to $|\nabla u|^{p-2} \nabla u$ strongly in $L^{1}(Q)$.

Proof. Estimates (5.4) are classical sine the work [BPR]. The a.e. convergence of $\left(u_{n}\right)$ can be proved using properties of truncations and the Marcinkiewicz estimates, see e.g., [BM, AA1]. The a.e convergence of $\left(\nabla u_{n}\right)$ is proved in [BDGO] when $b=1$ and the later technical points can be found in $[\mathrm{P}]$.

First of all, we need to show the existence of renormalized solution to (5.1). The proof which is based on the Theorem 4.1, is quite standard but we sketch it for completeness. 


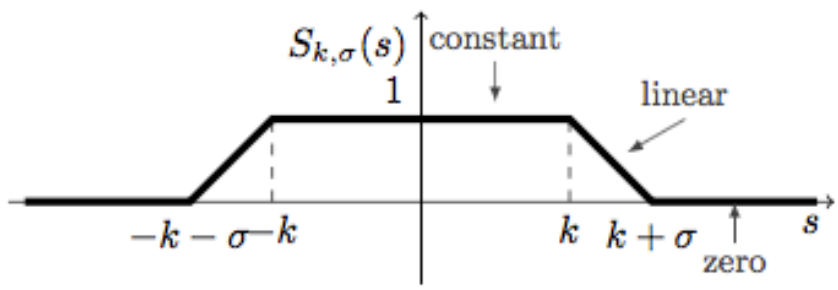

Figure 6. The function $S_{k, \sigma}(s)$

Proposition 5.4. Let a satisfies (2.1) - (2.3) and $u_{n}, h_{n}\left(u_{n}\right)$ and $\mu_{n}$ are defined as before. Then, for any fixed $n \in \mathbb{N}$, there exists a renormalized solution $u$ such that $(i)-(i v)$ holds true up to a subsequences and $u$ is a renormalized in the sense of Definition 5.1.

Sketch of the proof. Step 1. Basic estimates. Both sequences $\left(\mu_{n}\right)$ and $\left(h\left(u_{n}\right)\right)$ are bounded in $L^{1}(Q)$. Therefore, the solutions $u_{n}$ satisfy the estimates recalled in Proposition 5.3 and up to a subsequence $(i)-(i v)$ holds. Moreover, Proposition 5.2 applies and gives the estimate

$$
\operatorname{cap}_{p}\left\{\left|b\left(u_{n}\right)\right|>k\right\} \leq C \max \left\{k^{\frac{-1}{p}}, k^{\frac{-1}{p^{\prime}}}\right\} \quad \forall k \geq 1, \forall n \geq 1 .
$$

Finally, for $\sigma \geq 0$, consider the function $h_{\sigma}(s)=\frac{1}{\sigma}\left(T_{k+\sigma}(s)-T_{k}(s)\right)$, which is a piecewise inner odd function vanishing for $|s| \leq k$ and constant for $|s|>k+\sigma$. Let $\zeta_{\epsilon}(t)=1-\frac{1}{\epsilon} T_{\epsilon}(t-T+2 \epsilon)^{+}$, where $\epsilon \geq 0$; this function belongs to $W^{1, \infty}(0, T)$ and has compact support in $[0, T]$. By using $h_{\sigma}\left(u_{n}\right) \zeta_{\epsilon}$ as test function in (5.3) and letting $\epsilon \rightarrow 0$, we have

$$
\begin{aligned}
& \frac{1}{\sigma} \int_{\left\{k<\left|b\left(u_{n}\right)\right|<k+\sigma\right\}} a(t, x, u, \nabla u) \cdot \nabla u_{n} d x d t+\int_{Q} h_{n}\left(u_{n}\right) h_{\sigma}\left(u_{n}\right) d x d t \\
& \quad \leq \int_{Q} h_{\sigma}\left(b\left(u_{n}\right)\right) \mu_{n} d x d t+\int_{\left\{\left|b\left(u_{n}\right)\right|>k\right\}}\left|b\left(u_{0}^{n}\right)\right| d x
\end{aligned}
$$

which implies, in particular,

$$
\begin{aligned}
& \frac{1}{\sigma} \int_{\left\{k \leq\left|b\left(u_{n}\right)\right|<k+\sigma\right\}} a\left(t, x, u_{n}, \nabla u_{n}\right) \cdot \nabla u_{n} d x d t+\int_{\left\{\left|b\left(u_{n}\right)\right|>k\right\}}\left|h_{n}\left(u_{n}\right)\right| d x d t \\
& \leq \int_{\left\{\left|b\left(u_{n}\right)\right|>k\right\}}\left|u_{n}\right| d x d t+\int_{\left\{\left|b\left(u_{0}^{n}\right)\right|>k\right\}}\left|b\left(u_{0}^{n}\right)\right| d x
\end{aligned}
$$

and then

$$
\frac{1}{\sigma} \int_{\left\{k \leq\left|b\left(u_{n}\right)\right|<k+\sigma\right\}} a\left(t, x, u_{n}, \nabla u_{n}\right) \cdot \nabla u_{n} d x d t \leq C
$$

Step 2. Equation satisfied by truncations. For $\sigma>0$ small, consider the functions $S_{k, \sigma}(s)$ (see Figure 6) and $T_{k, \sigma}(s)$ given by

$$
S_{k, \sigma}(s)=\left\{\begin{array}{ll}
0 & \text { if }|s| \geq k+\sigma, \\
1 & \text { if }|s| \leq k, \\
\text { affine } & \text { otherwise, }
\end{array} \quad T_{k, \sigma}(s)=\int_{0}^{s} S_{k, \sigma}(\gamma) d \gamma\right.
$$

Recall that $T_{k, \sigma}(s)$ converges pointwise to $T_{k}(s)$ as $\sigma \rightarrow 0$. Given $\varphi \in C_{c}^{\infty}(Q)$, multiply the equation solved by $u_{n}$ by $S_{k, \sigma}\left(b\left(u_{n}\right)\right) \varphi$. We then have

$$
\begin{aligned}
& T_{k, \sigma}\left(b\left(u_{n}\right)\right)_{t}-\operatorname{div}\left(S_{k, \sigma}\left(b\left(u_{n}\right)\right) a\left(t, x, u_{n}, \nabla u_{n}\right)\right)+S_{k, \sigma}\left(b\left(u_{n}\right)\right) h_{n}\left(u_{n}\right) \\
& \quad=S_{k, \sigma}\left(b\left(u_{n}\right)\right) \mu_{n}+\frac{1}{\sigma} a\left(t, x, u_{n}, \nabla u_{n}\right) \cdot \nabla u_{n} \operatorname{sign}\left(b\left(u_{n}\right)\right) \chi_{\left\{k \leq\left|b\left(u_{n}\right)\right|<k+\sigma\right\}} \text { in } \mathcal{D}^{\prime}(Q) .
\end{aligned}
$$


Let

$$
\Lambda_{n, \sigma}^{k}=S_{k, \sigma}\left(b\left(u_{n}\right)\right)\left(\mu_{n}-h_{n}\left(u_{n}\right)\right)+\frac{1}{\sigma} a\left(t, x, u_{n}, \nabla u_{n}\right) \cdot \nabla u_{n} \operatorname{sign}\left(b\left(u_{n}\right)\right) \chi_{\left\{k<\left|b\left(u_{n}\right)\right|<k+\sigma\right\}}
$$

with this notation, we have

$$
\left(T_{k}\left(b\left(u_{n}\right)\right)\right)_{t}-\operatorname{div}\left(S_{k, \sigma}\left(b\left(u_{n}\right)\right) a\left(t, x, u_{n}, \nabla u_{n}\right)\right)=\lambda_{n, \sigma}^{k} \text { in } \mathcal{D}^{\prime}(Q) .
$$

Thanks to estimate (5.6), and since $\left|S_{k, \sigma}\right| \leq 1$, the functions $\Lambda_{n, \sigma}^{k}$ are bounded in $L^{1}(Q)$ uniformly with respect to $\sigma$ and $n$. Indeed, to be more precise, (5.6) and the definition of $S_{k, \sigma}$ imply the estimate

$$
\left\|\Lambda_{n, \sigma}^{k}\right\|_{L^{1}(Q)} \leq \int_{\left\{\left|b\left(u_{0}^{n}\right)\right|>k\right\}}\left|b\left(u_{0}^{n}\right)\right| d x+2\left(\int_{\left\{\left|b\left(u_{u}\right)\right|>k\right\}}\left|\mu_{n}\right| d x d t+\int_{\left\{\left|b\left(u_{u}\right)\right|>k\right\}}\left|h_{n}\left(u_{n}\right)\right| d x d t\right) .
$$

Step 3. Limit as $n$ goes to infinity. Applying Proposition 5.3 to the sequence $\left(u_{n}\right)$, there exists a function $u \in L^{1}(Q)$ such that $b(u) \in L^{\infty}\left(0, T ; L^{1}(\Omega)\right), T_{k}(b(u)) \in L^{p}\left(0, T ; W_{0}^{1, p}(\Omega)\right)$ for all $k>0$ and, up to a subsequence,

$$
u_{n} \rightarrow u, \quad b\left(u_{n}\right) \rightarrow b(u), \quad \nabla u_{n} \rightarrow \nabla u \text { almost everywhere in } Q .
$$

Let now $\left(\sigma_{n}\right)$ be a sequence of positive numbers converging to 0 , as $n \rightarrow \infty$. Using the definition of $S_{k, \sigma}(s)$, $\nabla u=0$ a.e. in $\{|b(u)=k|\}$ and $a(t, x, u, 0)=0$, we deduce that

$$
S_{k, \sigma}\left(b\left(u_{n}\right)\right) a\left(t, x, u_{n}, \nabla u_{n}\right) \rightarrow a\left(t, x, T_{k}(u), \nabla T_{k}(u)\right) \text { a.e. in } Q .
$$

Since $S_{k, \sigma_{n}}\left(b\left(u_{n}\right)\right) a\left(t, x, u_{n}, \nabla u_{n}\right)$ is bounded in $L^{p^{\prime}}(Q)$, it also follows that

$$
S_{k, \sigma_{n}}\left(b\left(u_{n}\right)\right) a\left(t, x, u_{n}, \nabla u_{n}\right) \rightarrow a\left(t, x, T_{k}(u), \nabla T_{k}(u)\right) \text { weakly in } L^{p^{\prime}}(Q) .
$$

By properties of convolution, clearly we have $\mu_{n} \rightarrow^{*} \mu$ in the weak topology of measures. Finally, since the sequence $\left(\Lambda_{n, \sigma_{n}}^{k}\right)$ is bounded in $L^{1}(Q)$, there exists a bounded measure $\lambda_{k}$ such that, up to a subsequence, $\left(\Lambda_{n, \sigma_{n}}^{k}\right)_{n}$ converges to $\lambda_{k}$ in the weak ${ }^{*}$ topology of $(C(\bar{Q}))^{\prime}$. We deduce from (5.8) that $u$ satisfies

$$
\begin{aligned}
& -\int_{Q} T_{k}(b(u)) \varphi_{t} d x d t+\int_{Q} a\left(t, x, T_{k}(u), \nabla T_{k}(u)\right) . \nabla \varphi d x d t+\int_{Q} h(u) \varphi d x d t \\
& =\int_{Q} \varphi d \mu+\int_{Q} \varphi d \lambda_{k}+\int_{Q} T_{k}\left(b\left(u_{0}\right)\right) \varphi(0, x) d x
\end{aligned}
$$

for every $\varphi \in C_{c}^{\infty}([0, T] \times \Omega)$.

Step 4. Estimate of $\lambda_{k}$. By weak* lower semi-continuity of the norm, we have

$$
\left\|\Lambda_{k}\right\|_{\mathcal{M}_{0}(Q)} \leq \liminf _{n \rightarrow \infty}\left\|\Lambda_{n, \sigma_{n}}^{k}\right\|_{\mathcal{M}_{0}(Q)} .
$$

Since, by Proposition 2.1, the sequence $\left(\mu_{n}\right)$ is equidiffuse, thanks to the uniform estimate (5.5), we deduce that

$$
\limsup _{k \rightarrow \infty} \int_{\left\{\left|b\left(u_{n}\right)\right|>k\right\}}\left|\mu_{n}\right| d x d t=0 .
$$

By the equi-integrability of $\left(b\left(u_{0}^{n}\right)\right)$ and $\left(h\left(u_{n}\right)\right)$, the two terms with $h\left(u_{n}\right)$ and $b\left(u_{0}^{n}\right)$ satisfy

$$
\limsup _{k \rightarrow \infty} \int_{\left\{\left|b\left(u_{n}\right)\right|>k\right\}}\left|h_{n}\left(u_{n}\right)\right| d x d t=0, \quad \limsup _{k \rightarrow \infty} \int_{\left\{\left|b\left(u_{n}\right)\right|>k\right\}}\left|b\left(u_{0}^{n}\right)\right| d x d t=0 .
$$

Then by (5.9), we have

$$
\left\|\lambda_{k}\right\|_{\mathcal{M}_{0}(Q)} \leq \liminf _{k \rightarrow \infty}\left\|\Lambda_{n, \sigma_{n}}^{k}\right\|_{\mathcal{M}_{0}(Q)} \leq \epsilon_{k} \rightarrow 0 .
$$

We conclude that $\lambda_{k}$ satisfies (4.2), and hence, $u$ is a renormalized solution. 
5.2. Uniqueness of renormalized solutions. As far as the notion of renormalized solution of (5.1) is concerned, we follow the result of [AA2]. Namely, a modification of $L^{1}$-contraction principle of [AA2, Theorem 6.1] replacing $\mu$ with $\mu-h(u)$ as right-hand side.

Lemma 5.1. Let $u_{1}, u_{2}$ be two renormalized solutions of problem (5.1) with data $\left(b\left(u_{0}^{1}\right), \mu_{1}\right)$ and $\left(b\left(u_{0}^{2}\right), \mu_{2}\right)$ respectively. Then,

$\int_{\Omega}\left(b\left(u_{1}\right)-b\left(u_{2}\right)\right)^{+}(\tau) d x+\int_{0}^{\tau} \int_{\Omega}\left(h\left(u_{1}-h\left(u_{2}\right)\right) \operatorname{sign}^{+}\left(b\left(u_{1}\right)-b\left(u_{2}\right)\right) d x d t \leq \|\left(b\left(u_{0}^{1}\right)-b\left(u_{0}^{2}\right)\left\|_{L^{1}(\Omega)}+\right\|\left(\mu_{1}-\mu_{2}\right)^{+} \|_{\mathcal{M}_{0}(Q)}\right.\right.$

for almost every $\tau \in(0, T)$.

We conclude by the following result

Theorem 5.2. Let $h$ be nonincreaing, then there is at most one renormalized solution to (5.1).

Proof. The uniqueness of solution in (5.1) can be proved in the lines of the papers [PPP2, AA2].

Acknowledgments The authors would like to thank the editor and anonymous referees who kindly reviewed the earlier version of this manuscript.

\section{References}

[AA1] M. Abdellaoui, E. Azroul, Renormalized solutions for nonlinear parabolic equations with general measure data, Electron. J. Differential Equations, Vol. 2018, No. 132, pp. 1-21.

[AA2] M. Abdellaoui, E. Azroul, Nonlinear parabolic equations with soft measure data, arXive:submit $\backslash 2570936$ Submitted.

[AA3] M. Abdellaoui, E. Azroul, H. Redwane, Nonlinear parabolic equations of porous medium type with unbounded term and general measure data, Submitted.

[ABR] A. Aberqi, J. Bennouna, H. Redwane, Hicham, A nonlinear parabolic problems with lower order terms and measure data, Thai Journal of Mathematics, Vol. 14, No. 1 (2016), 115-130.

[AR] K. Ammar, Hicham Redwane, Existence of positive solutions for a class of parabolic equations with natural growth terms and $L^{1}$ data, Acta Mathematica Scientia, Vol. 34, No. 4 (2014), 1127-1144.

[B6] P. Bénilan, L. Boccardo, T. Gallouët, R. Gariepy, M. Pierre, J.-L. Vazquez, An $L^{1}$-theory of existence and uniqueness of solutions of nonlinear elliptic equations, Ann Scuolo Norm. Sup. Pisa, 22 no. 2 (1995), pp. 240-273.

[BCW] P. Bénilan, J. Carrillo, P. Wittbold, Renormalized entropy solutions of scalar conservation laws, Ann. Scuola Norm. Sup. Pisa Cl. Sci. (4)29 (2000), 313-327.

[BDGO] L. Boccardo, A. Dall'Aglio, T. Gallouët, L. Orsina Nonlinear parabolic equations with measure data, Journal of Functional Analysis 147 (1997), 237-258.

[BF] D. Blanchard, G. Francfort, A few results on a class of degenerate parabolic equations, Ann. SCM. Norm. Sup. Piss, 8(2), 1991, 213-249.

[BG] L. Boccardo and T. Gallouët, Nonlinear elliptic and parabolic equations involving measure data, J. Funct. Anal. 87 (1989), 149-169.

[BGR] D. Blanchard, O. Guibé, H. Redwane, Existence and uniqueness of a solution for a class of parabolic equations with two unbounded nonlinearities, Communications on Pure and Applied Analysis, Vol. 15, No. 1, January 2016, 197-217.

[BK] M. Bendahmane, K. H. Karlsen. Renormalized solutions of an anisotropic reaction-diffusion-advection system with $L^{1}$ data. Communications on Pure \& Applied Analysis, 5(4) (2006), 733-762.

[BM] D. Blanchard, F. Murat, Renormalized solutions of nonlinear parabolic problems with $L^{1}$ data, existence and uniqueness, Proc. of the Royal Soc. of Edinburgh Section A127 (1997), 1137-1152.

[BP1] D. Blanchard, A. Porretta, Nonlinear parabolic equations with natural growth terms and measure initial data, Annali della Scuola Normale Superiore di Pisa, Classe di Scienze 4e série, tome 30, no 3-4 (2001), p. $583-622$ 
[BP2] D. Blanchard, A. Porretta, Stefan problems with nonlinear diffusion and convection, J. Differential Equations 210 (2005), no. 2, 383-428.

[BPR] D. Blanchard, F. Petitta, H. Redwane, Renormalized solutions of nonlinear parabolic equations with diffuse measure data, Manuscripta Math., 141, no. 3-4 (2013), 601-635.

[BR1] D. Blanchard, H. Redwane, Renormalized solutions for a class of nonlinear evolution problems, J. Math. Pures Appl. (9)77, 1998, 117-151.

[BR2] D. Blanchard, H. Redwane, Existence of a solution for a class of parabolic equations with three unbounded nonlinearities, natural growth terms and $L^{1}$ data, Arab Journal of Mathematical Sciences, Vol. 20, No. 2 (2014), 157-176,

[BRM] A. Bouajaja, H. Redwane, A. Marah, Existence and Uniqueness of Renormalized Solutions to Nonlinear Parabolic Equations with Lower Order Term and Diffuse Measure Data, A. Mediterr. J. Math. (2018) 15: 178.

[DMOP] G. Dal Maso, F. Murat, L. Orsina, A. Prignet, Renormalized solutions of elliptic equations with general measure data, Ann. Scuola Norm. Sup. Pisa Cl. Sci., 28 (1999), 741-808.

[DP] J. Droniou, A. Prignet, Equivalence between entropy and renormalized solutions for parabolic equations with smooth measure data, No DEA 14 (2007), no. 1-2, 181-205.

[DPP] J. Droniou, A. Porretta, A. Prignet, Parabolic Capacity and soft measures for nonlinear equations, Potential Analysis, Vol 19, No 2 (2003), pp. 99-161.

[Eg] D. Egorov, Sur les suites des fonctions mesurables, Comptes Rendus Acad. Sci. Paris, 152 (1911), $244-246$.

[FST] M. Fukushima, K. Sato and S. Taniguchi. On the closable part of pre-Dirichlet forms and the finitene supports of underlying measures. Osaka J. Math. 28 (1991), 517-535.

[HKM] J. Heinonen, T. Kilpelainen and O. Martio, Nonlinear Potential Theory of Degenerate Elliptic Equations, Oxford University Press, Oxford, 1993.

[GM] T. Gallouët, J. M. Morel, Resolution of a semilinear equation in $L^{1}$, Proc. Roy. Soc. Edinburgh Sect. A96 (1984), 275-288.

[GSZW] Z. C. Guo, J. B. Sun, D. Z. Zhang, B. Y. Wu, Adaptive Perona-Malik model based on the variable exponent for image denoising, IEEE Transactions on Image Processing, 21 (2012), 958-967.

[KR] Klimsiak, T., Rozkosz, A.: On the structure of diffuse measures for parabolic capacities, arXiv :1808.06422v2 [math.AP] 18 (Feb 2019)

[L1] J.-L. Lions, Quelques méthodes de résolution des problèmes aux limites non linéaire, Dunod et GauthierVillars, (1969).

[L2] P.-L. Lions, Mathematical Topics in Fluid Mechanics, Vol. 1: Incompressible models, The Clarendon Press, Oxford University Press, New York, 1996.

[MR1] A. Marah, H. Redwane, Nonlinear parabolic equations with diffuse measure data, Journal of Nonlinear Evolution Equations and Applications, 3 (2017), 27-48.

[MR2] A. Marah, H. Redwane, Existence of a renormalized solution of nonlinear parabolic equations with general measure data, submitted.

[P] A. Porretta, Existence results for nonlinear parabolic equations via strong convergence of truncations, Ann. Mat. Pura ed Appl. (IV), 177 (1999), 143-172.

[Pe1] F. Petitta, Renormalized solutions of nonlinear parabolic equations with general measure data, Annali di Matematica (2008), 187-563.

[PP] F. Petitta, A. Porretta, On the notion of renormalized solution to nonlinear parabolic equations with general measure data, Journal of Elliptic and Parabolic Equations, 1 (2015), 201-214.

[Pe3] F. Petitta, Asymptotic behavior of solutions for linear parabolic equations with general measure data, C. R. Acad. Sci. Paris, Ser. I 344 (2007): 571-576.

[PPP1] F. Petitta, A. C. Ponce, A. Porretta, Approximation of diffuse measures for parabolic capacities, C. R. Acad. Sci. Paris, Ser. I 346 (2008), 161-166.

[PPP2] F. Petitta, A. C. Ponce, A. Porretta, Diffuse measures and nonlinear parabolic equations, Journal of Evolution Equations, 11 (2011), no. 4, 861-905.

[Pr1] A. Prignet, Remarks on existence and uniqueness of solutions of elliptic problems with right hand side measures, Rend. Mat., 15 (1995), 321-337. 
[Pr2] A. Prignet, Existence and uniqueness of entropy solutions of parabolic problems with $L^{1}$ data. Nonlinear. Anal. TMA 28, 1943-1954 (1997).

[S] G. Stampacchia, Le problème de Dirichlet pour les équations elliptiques du seconde ordre à coefficientes discontinus, Ann. Inst. Fourier (Grenoble), 15 (1965), 189-258.

[Se] J. Serrin. Pathological solutions of elliptic differential equations. Ann. Scuola Norm. Sup. Pisa Cl. Sci. 18, 385-387 (1964).

[V] J. L. Vázquez, The porous medium equation, Mathematical theory. Oxford Math. Monographs. Oxford Univ. Press, 2007. 